\title{
Character Polynomials, their q-analogs and the Kronecker product
}

\author{
A. M. Garsia* and A. Goupil ** \\ Department of Mathematics \\ University of California, San Diego, California, USA \\ garsia@math.ucsd.edu \\ Département de mathématiques et d'informatique \\ Université du Québec à Trois-Rivières, Trois-Rivières, Québec, Canada \\ alain.goupil@uqtr.ca
}

Submitted: Sep 22, 2008; Accepted: Jul 25, 2009; Published: Jul 31, 2009

Mathematics Subject Classifications: 20C30, 20C08, 05E05, 05A18, 05A15

Dedicated to Anders Björner on the occasion of his sixtieth birthday.

\begin{abstract}
The numerical calculation of character values as well as Kronecker coefficients can efficently be carried out by means of character polynomials. Yet these polynomials do not seem to have been given a proper role in present day literature or software. To show their remarkable simplicity we give here an "umbral" version and a recursive combinatorial construction. We also show that these polynomials have a natural counterpart in the standard Hecke algebra $\mathcal{H}_{n}(q)$. Their relation to Kronecker products is brought to the fore, as well as special cases and applications. This paper may also be used as a tutorial for working with character polynomials in the computation of Kronecker coefficients.
\end{abstract}

\section{Introduction}

We recall that the value $\chi_{\alpha}^{\lambda}$ of the irreducible $S_{n}$ character indexed by a partition $\lambda=\left(\lambda_{1}, \ldots, \lambda_{k}\right)$ at a permutation of $S_{n}$ with cycle structure $\alpha=1^{a_{1}} 2^{a_{2}} \cdots n^{a_{n}}$ is given by the Frobenius formula

$$
\chi_{\alpha}^{\lambda}=\left.\Delta(x) p_{\alpha}\right|_{x_{1}^{\lambda_{1}+n-1} x_{2}^{\lambda_{2}+n-2} \cdots x_{n}^{\lambda_{n}+n-n}}
$$

* Work supported by a grant from NSF.

** Work partially supported by a grant from NSERC.

THE ELECTRONiC JOURNAL OF COMBINATORICS 16(2) (2009), \#R19 
where $\Delta(x)=\Delta\left(x_{1}, \ldots, x_{n}\right)$ and $p_{\alpha}=p_{\alpha}\left(x_{1}, \ldots, x_{n}\right)$ denote respectively the Vandermonde determinant and the power sums symmetric functions. The character polynomial $q_{\mu}\left(x_{1}, x_{2}, \ldots, x_{n}\right)$ is the unique polynomial in $\mathbb{Q}\left[x_{1}, x_{2}, \ldots, x_{n}\right]$ with the property that for all partitions $\mu \vdash k$ and $\lambda=(n-k, \mu)$ with $n-k \geq \mu_{1}$ we have

$$
\chi_{1^{a_{1} 2^{a_{2}} \ldots n^{a_{n}}}}^{(n-k, \mu)}=q_{\mu}\left(a_{1}, a_{2}, \ldots, a_{n}\right) .
$$

Moreover, with an appropriate change of sign and rearrangements of the parts of ( $n-$ $k, \mu)$ this can be shown to remain true even when $n-k<\mu_{1}$. The simplest case of equation I.2 is the well known formula

$$
q_{1}(x)=x_{1}-1
$$

which implies that for all $n \geq 2$ the value of the character $\chi^{(n-1,1)}$ at a permutation $\sigma \in S_{n}$ is simply equal to one less than the number $x_{1}$ of fixed points of $\sigma$. Character polynomials were implicitly used for the first time in the work of Murnaghan $[\mathrm{Mu}]$ and were identified as such later by Specht in [Sp] where a determinantal form for character polynomials and a proof of equation I.2 are given. Treatments of character polynomials vary from the purely existential as in Kerber [Ke], to the very explicit as in Macdonald's book ([Ma] ex. I.7.13 and I.7.14). What is quite surprising, as we see in I.3, is how little information from the cycle structure may be needed to compute the whole sequence of character values $\chi^{(n-k, \mu)}$. We will show here that a slight addition to the computation carried out in [Ma] yields a formula of utmost simplicity which brings to explicit evidence this minimal dependence on the cycle structure.

To state our formula let us denote by " $\downarrow$ " the "umbral" operator that transforms a monomial into a product of lower factorial polynomials. To be precise we set

$$
\downarrow x^{a}=\downarrow x_{1}^{a_{1}} x_{2}^{a_{2}} \cdots x_{m}^{a_{m}}=\left(x_{1}\right)_{a_{1}}\left(x_{2}\right)_{a_{2}} \cdots\left(x_{m}\right)_{a_{m}}
$$

with $(x)_{a}=x(x-1)(x-2) \cdots(x-a+1)$. This given we have

Proposition I.1 For all $\mu \vdash k$, the character polynomial $q_{\mu}$ depends at most on the first $k$ variables. More precisely

$$
q_{\mu}\left(x_{1}, x_{2}, \cdots, x_{k}\right)=\downarrow \sum_{\alpha \vdash k} \frac{\chi_{\alpha}^{\mu}}{z_{\alpha}} \prod_{i=1}^{k}\left(i x_{i}-1\right)^{a_{i}}
$$

where $z_{\alpha}=1^{a_{1}} 2^{a_{2}} \cdots k^{a_{k}} a_{1} ! a_{2} ! \cdots a_{k}$ !. In other words, equation I.5 states that we obtain any character polynomial $q_{\mu}$ by the following easy sequential steps: 
a) Expand the Schur function $s_{\mu}$ in the power sums basis : $s_{\mu}=\sum_{\alpha \vdash k} \frac{\chi_{\alpha}^{\mu}}{z_{\alpha}} p_{\alpha}$.

b) Replace each power sum $p_{i}$ by $i x_{i}-1$.

c) Expand each product $\prod_{i}\left(i x_{i}-1\right)^{a_{i}}$ as a sum $\sum_{g} c_{g} \prod_{i} x_{i}^{g_{i}}$.

d) Replace each $x_{i}^{g_{i}}$ by $\left(x_{i}\right)_{g_{i}}$.

Let us compute the polynomial $q_{(3)}\left(x_{1}, x_{2}, x_{3}\right)$ by using the preceding steps :

a) $s_{3}=\frac{1}{6}\left(p_{1^{3}}+3 p_{(21)}+2 p_{(3)}\right)$

b) $\frac{1}{6}\left(p_{1^{3}}+3 p_{(21)}+2 p_{(3)}\right) \rightarrow \frac{1}{6}\left(\left(x_{1}-1\right)^{3}+3\left(2 x_{2}-1\right)\left(x_{1}-1\right)+2\left(3 x_{3}-1\right)\right)$

c) $\frac{1}{6}\left(\left(x_{1}-1\right)^{3}+3\left(2 x_{2}-1\right)\left(x_{1}-1\right)+2\left(3 x_{3}-1\right)\right)=\frac{1}{6} x_{1}^{3}-\frac{1}{2} x_{1}^{2}+x_{1} x_{2}-x_{2}+x_{3}$

d) $q_{(3)}\left(x_{1}, x_{2}, x_{3}\right)=\frac{1}{6}\left(x_{1}\right)_{3}-\frac{1}{2}\left(x_{1}\right)_{2}+x_{1} x_{2}-x_{2}+x_{3}$

Note that when we set $x_{1}=n$ in $q_{\mu}$ and $x_{i}=0$ for all $i>1$, we obtain the number $f^{(n-k, \mu)}$ of standard tableaux of shape $(n-k, \mu)$. In view of the classical hook formula, this must reduce to the identity

$$
f^{(n-k, \mu)}=\left.q_{\mu}\left(x_{1}, 0,0, \ldots\right)\right|_{x_{1}=n}=\left.\frac{\left(x_{1}\right)_{k+\mu_{1}} f^{\mu} / k !}{\prod_{i=1}^{\mu_{1}}\left(x_{1}-k+\mu_{i}^{\prime}-i+1\right)}\right|_{x_{1}=n}
$$

$\forall \mu \vdash k \& n-k \geq \mu_{1}$, where $\mu^{\prime}=\left(\mu_{1}^{\prime}, \mu_{2}^{\prime}, \ldots\right)$ is the conjugate partition of $\mu$.

An immediate consequence of equation I.5 is a recursive algorithm for the construction of the character polynomials that does not directly involve any of the symmetric group characters.

Corollary I.1 For a given partition $\mu \vdash k$, let $\tilde{\mu}$ denote the partition obtained by removing the first part from $\mu$. Then

$$
q_{\mu}\left(x_{1}, x_{2}, \cdots, x_{k}\right)=\downarrow \sum_{1 a_{1}+2 a_{2}+\cdots+k a_{k}=k} \frac{q_{\tilde{\mu}}\left(a_{1}, a_{2}, \ldots, a_{k}\right)}{1^{a_{1}} 2^{a_{2}} \cdots k^{a_{k}} a_{1} ! a_{2} ! \cdots a_{k} !} \prod_{i=1}^{k}\left(i x_{i}-1\right)^{a_{i}}
$$

with initial setting

$$
q_{\emptyset}\left(a_{1}, a_{2}, \ldots, a_{k}\right)=1 \text {. }
$$

Our next result is a combinatorial formula for the character polynomials which has some kinship with the Murnaghan-Nakayama rule, and is quite suitable for hand calculations.

Theorem I.1 For a given $\mu \vdash k$, let $b d(i, \mu)$ be the maximal number of border strips of length $i$ that can successively be removed from the diagram of $\mu$ so that a Ferrers diagram remains. Then the terms in $q_{\mu}$ that contain the variable $x_{i}, 2 \leq i \leq k$ but no variable $x_{r}$ with $r>i$ is given by the recursive rule

$$
\begin{aligned}
q_{\mu}\left(x_{1}, \ldots, x_{i}, 0, \ldots\right)-q_{\mu}\left(x_{1}, \ldots, x_{i-1}, 0, \ldots\right) \\
=\sum_{j=1}^{b d(i, \mu)}\left(\begin{array}{c}
x_{i} \\
j
\end{array}\right) \sum_{S=\left(\mu^{0}, \mu^{1}, \ldots, \mu^{j}\right)}(-1)^{h t(S)} q_{\mu^{j}}\left(x_{1}, \ldots, x_{i-1}, 0\right)
\end{aligned}
$$


where the inner sum is over all $(j+1)$-tuples $S$ of partitions $\mu^{r}$ such that each $\mu^{r}-\mu^{r+1}$ is a border strip of length $i$ and $h t(S)=\sum_{r=0}^{j-1}\left(h e i g h t\left(\mu^{r}-\mu^{r+1}\right)-1\right)$. The initial term $q_{\mu}\left(x_{1}, 0, \ldots\right)$ is computed via equation I.6.

For instance, the polynomial $q_{(3,1,1)}\left(x_{1}, x_{2}, \ldots, x_{5}\right)$ is recursively constructed as follows.

$$
\begin{aligned}
q_{(3,1,1)}\left(x_{1}, \ldots, x_{5}\right)= & q_{(3,1,1)}\left(x_{1}\right)+(-1)^{0}\left(\begin{array}{c}
x_{2} \\
1
\end{array}\right) q_{\left(1^{3}\right)}\left(x_{1}\right)+(-1)^{1}\left(\begin{array}{c}
x_{2} \\
1
\end{array}\right) q_{(3)}\left(x_{1}\right) \\
& +(-1)^{1} 2\left(\begin{array}{c}
x_{2} \\
2
\end{array}\right) q_{(1)}\left(x_{1}\right)+(-1)^{2}\left(\begin{array}{c}
x_{5} \\
1
\end{array}\right) q_{\emptyset}\left(x_{1}, \ldots, x_{4}\right) \\
= & \frac{x_{1}\left(x_{1}-1\right) \cdots\left(x_{1}-7\right)}{\left(x_{1}-2\right)\left(x_{1}-5\right)\left(x_{1}-6\right)} \frac{f^{(3,1,1)}}{5 !}+x_{2}\left(\begin{array}{c}
x_{1}-1 \\
3
\end{array}\right) \\
& \quad-x_{2}\left(\frac{x_{1}\left(x_{1}-1\right) \cdots\left(x_{1}-5\right)}{\left(x_{1}-2\right)\left(x_{1}-3\right)\left(x_{1}-4\right)} \frac{f^{(3)}}{3 !}\right)-2\left(\begin{array}{c}
x_{2} \\
2
\end{array}\right)\left(x_{1}-1\right)+x_{5}
\end{aligned}
$$

where the last equality follows from equation I.6.

This paper is organized as follows. In the first section we introduce our notation, make some definitions and prove some auxiliary facts. In the second section we treat the classical $S_{n}$ case, prove our umbral formula for the character polynomials as well as Theorem I.1. In the third section, striving to make our writing accessible to a wider audience, we give a brief tutorial on Kronecker products including simple proofs of some basic results of the theory. The experts in symmetric function theory may skip this section. In the fourth section we use the pairing $s_{\mu} \rightarrow q_{\mu}$ to define a degree preserving isomorphism that sends the vector space $\Lambda$ of symmetric polynomials onto the vector space of polynomials $\mathbb{Q}\left[x_{1}, x_{2}, x_{3}, \ldots\right]$. We then use this map to derive some well known and some not so well known properties of Kronecker products. The study of this map leads to another family of polynomials that we call "set polynomials " which enjoy properties akin to those of character polynomials and can also be used to compute Kronecker products. In the fifth section we treat the Hecke algebra case and derive our $q$-analogs of character polynomials. We present comparative tables of character and q-character polynomials. In the sixth and last section we explore some consequences of our techniques and give some applications. In particular we obtain an explicit formula yielding a generating function for the occurence of $s_{(n)}$ in Kronecker powers of $h_{r} h_{n-r}$ for every fixed $r \geq 1$. This generating function may be viewed as a solution to a problem first formulated by Comtet in [Co], namely the enumeration of coverings of a set of cardinality $n$ by sets of cardinality $r$. The corresponding generating function for $r=2$ was first given by Labelle in [La]. A surprisingly simple argument yields the general result and in particular the Labelle result. The calculation of character polynomials also yield 
unexpected results. For instance, it comes out that the polynomial $\sum_{s=0}^{k}(-1)^{k-s} n(n-$ 1) $\cdots(n-s+1)$ enumerates, for $n \geq 2 k$, the number of permutations $\sigma \in S_{n}$ with longest increasing subsequence $\sigma(1), \sigma(2), \cdots, \sigma(n-k)=n$. A direct proof of this makes an amusing combinatorial exercise. A second unexpected outcome is a formula for the well known Bell numbers that does not seem to appear in the literature. We terminate the paper with the computation of certain remarkable character polynomials.

Acknowledgement. The authors wish to thank Alain Lascoux for his helpful suggestions and remarks on an earlier version of this paper. We also thank the referees for their excellent review.

\section{Definitions and basic concepts}

To begin it will be convenient to write a partition $\alpha$ of $n$ as a weakly decreasing list of parts $\alpha=\left(\alpha_{1} \geq \alpha_{2} \geq \ldots \alpha_{k} \geq 1\right)$ or by giving the list of multiplicities of its parts : $\alpha=1^{a_{1}} 2^{a_{2}} \ldots n^{a_{n}}$. We will use greek letters $\lambda, \mu, \alpha, \ldots$ for the partitions and their parts and the corresponding roman letters $\ell_{i}, m_{i}, a_{i}$ for their multiplicities. The number $k$ of parts of a partition $\alpha=\left(\alpha_{1}, \alpha_{2}, \ldots, \alpha_{k}\right)$ is called the length of $\alpha$ and is denoted $\ell(\alpha)$. The weight $|\alpha|$ of a partition $\alpha$ is the sum of its parts and we extend this convention to any vector $a=\left(a_{1}, \ldots, a_{k}\right)$. The expression $z_{\alpha}=1^{a_{1}} 2^{a_{2}} \cdots n^{a_{n}} a_{1} ! \cdots a_{n}$ ! will be used throughout the text. Thus for a partition $\alpha$ of $n$ we will use the notations

$$
\begin{gathered}
\alpha=\left(\alpha_{1}, \alpha_{2}, \ldots, \alpha_{k}\right)=1^{a_{1}} 2^{a_{2}} \ldots n^{a_{n}}, \alpha \vdash n,|\alpha|=\|a\|=1 a_{1}+2 a_{2}+\cdots+n a_{n}=n ; \\
\ell(\alpha)=k=|a|=a_{1}+a_{2}+\ldots+a_{n} .
\end{gathered}
$$

We will need to merge partitions and for $\alpha=1^{a_{1}} 2^{a_{2}} \ldots n^{a_{n}}, \beta=1^{b_{1}} 2^{b_{2}} \ldots m^{b_{m}}$ and $n \geq m$ we use the operation $\alpha \vee \beta=1^{a_{1}+b_{1}} 2^{a_{2}+b_{2}} \ldots n^{a_{n}+b_{n}}$. As customary if $\mu$ is a partition, $\mu^{\prime}$ will denote the conjugate partition. It will also be convenient to denote by $\Lambda$, the space of symmetric functions and by $\Lambda^{=k}$ the subspace of symmetric functions that are homogeneous of degree $k$. The number of variables in a symmetric function will always be assumed to be greater or equal to its degree. The Hall scalar product of symmetric functions, with respect to which the Schur functions form an orthonormal system, will be denoted $\langle$,$\rangle .$

In this paper we shall make extensive use of plethystic notation. The first author has been a proponent of this device since the early 1980's after Lascoux showed that many identities in Macdonald's book could acquire a remarkable simplicity in terms of it. This notwithstanding, its use doesn't seem to have yet achieved widespread acceptance. This work will give us one more opportunity to show the power of this 
notational device in the theory of symmetric functions. To this end we recall that the plethystic substitution of a formal power series $E\left(t_{1}, t_{2}, \ldots, t_{s}, \ldots\right)$ into a symmetric polynomial $A$, denoted " $A[E]$ " is obtained by setting

$$
A[E]=\left.Q_{A}\left(p_{1}, p_{2}, \ldots\right)\right|_{p_{k} \rightarrow E\left(t_{1}^{k}, t_{2}^{k}, \ldots\right)}
$$

where $Q_{A}\left(p_{1}, p_{2}, \ldots\right)$ is the polynomial that gives the expansion of $A$ in terms of the power basis $\left\{p_{\alpha}\right\}_{\alpha}$. This given, if we let

$$
\Omega[X]=\exp \left(\sum_{k \geq 1} \frac{p_{k}[X]}{k}\right)
$$

then setting $E=z X_{n}$ with $X_{n}=\sum_{i=1}^{n} x_{i}$ we get

$$
\Omega\left[z X_{n}\right]=\prod_{i=1}^{n} \frac{1}{\left(1-z x_{i}\right)}=\sum_{m \geq 0} h_{m}\left[X_{n}\right] z^{m}=\exp \left(\sum_{k \geq 1} \frac{p_{k}\left[X_{n}\right]}{k} z^{k}\right)
$$

which is the generating function of the so-called "homogeneous" symmetric functions $h_{m}$. We should note that contrary to intuition the definition in 1.2 yields that

$$
p_{k}\left[-X_{n}\right]=p_{k}\left[-\sum_{i=1}^{n} x_{i}\right]=-\sum_{i=1}^{n} x_{i}^{k}=-p_{k}\left[X_{n}\right]
$$

This paradox, in spite of being a hindrance, is an asset. In fact, we need only distinguish the "plethystic" minus sign from the customary minus sign. This is easily achieved. When we want to replace a variable $x_{i}$ or a formal power series $E$ by its negative in the customary sense we simply prepend a minus sign to it as in " ${ }^{-} x_{i}$ " or " $-E$ " and use the regular minus sign for plethystic subtraction. Note that with this convention, and $X=\sum_{i} x_{i}$ we get

$$
p_{k}\left[-{ }^{-} X\right]=(-1)^{k-1} p_{k}[X]=\omega p_{k}[X]
$$

where " $\omega$ " is the fundamental involution on $\Lambda$ that sends the homogeneous basis onto the elementary basis. In particular from 1.4 we derive that for any symmetric funtion $A[X]$ we have, when the alphabet $X$ has a sufficient number of variables, $\omega A[X]=A\left[-^{-} X\right]$. Moreover, when $A$ is homogeneous of degree $k$, we see that this is equivalent to

$$
A[-X]=(-1)^{k} \omega A[X]
$$


In the same vein we show that

Proposition 1.1 For any partition $\lambda$ and any two alphabets $X=\sum_{i} x_{i}$ and $Y=\sum_{j} y_{j}$ we have the Schur function identity

$$
s_{\lambda}[X-Y]=\sum_{\mu \subseteq \lambda} s_{\lambda / \mu}[X](-1)^{|\mu|} s_{\mu^{\prime}}[Y] .
$$

In particular for any $m \geq 1$ we get that

$$
h_{m}[X-Y]=\sum_{k=0}^{m} h_{m-k}[X](-1)^{k} e_{k}[Y]
$$

and for $Y=1$ this specializes to

$$
h_{m}[X-1]=h_{m}[X]-h_{m-1}[X]
$$

Proof. Since 1.7 follows from 1.6 by setting $\lambda=(m)$ we need only show 1.6 . On the other hand 1.6 is an immediate consequence of 1.5 and of the addition formula ([Ma], ch. I, 5.9)

$$
s_{\lambda}[X+Y]=\sum_{\mu \subseteq \lambda} s_{\lambda / \mu}[X] s_{\mu}[Y] .
$$

upon replacement of $Y$ by $-Y$. In fact, from 1.5 it follows that

$$
s_{\mu}[-Y]=(-1)^{|\mu|} s_{\mu^{\prime}}[Y] .
$$

Alternatively we can prove 1.7 without using the addition formula. Indeed we have

$$
\begin{aligned}
\Omega[t(X-Y)] & =\exp \left(\sum_{k \geq 1} \frac{t^{k} p_{k}[X-Y]}{k}\right)=\exp \left(\sum_{k \geq 1} \frac{t^{k} p_{k}[X]}{k}\right) \exp \left(\sum_{k \geq 1} \frac{-t^{k} p_{k}[Y]}{k}\right) \\
& =\left(\sum_{a \geq 0} t^{a} h_{a}[X]\right)\left(\sum_{b \geq 0}(-t)^{b} e_{b}[Y]\right)=\sum_{n \geq 0} t^{n} \sum_{k=0}^{n} h_{k}[X](-1)^{n-k} e_{n-k}[Y]
\end{aligned}
$$

and since 1.3 gives $\Omega[t(X-Y)]=\sum_{n \geq 0} t^{n} h_{n}[X-Y]$, we obtain 1.7 by taking the coefficient of $t^{n}$.

More generally, for any formal power series $E$ we define, the "translation by $E$ " operator, " $\mathcal{T}_{E}$ ", on symmetric functions $A[X]$ by

$$
\mathcal{T}_{E} A[X]=A[X+E]
$$


Now for any symmetric function $A$ it is customary to denote by $A^{\perp}$ the adjoint of the operator multiplication by $A$, with respect to the Hall scalar product. It follows from the definition of skew Schur functions $s_{\lambda / \mu}$ that we may write $s_{\lambda / \mu}=s_{\mu}^{\perp} s_{\lambda}$. Thus 1.6 and 1.9 may be written in the form

$$
\mathcal{T}_{-Y} s_{\lambda}[X]=\left(\sum_{\mu}(-1)^{|\mu|} s_{\mu^{\prime}}[Y] s_{\mu}^{\perp}\right) s_{\lambda}[X] \quad \text { and } \quad \mathcal{T}_{Y} s_{\lambda}[X]=\left(\sum_{\mu} s_{\mu}[Y] s_{\mu}^{\perp}\right) s_{\lambda}[X]
$$

and since Schur functions form a basis, it follows that we may write

$$
\mathcal{T}_{-Y}=\sum_{\mu}(-1)^{|\mu|} s_{\mu^{\prime}}[Y] s_{\mu}^{\perp} \quad \text { and } \quad \mathcal{T}_{Y}=\sum_{\mu} s_{\mu}[Y] s_{\mu}^{\perp}
$$

Since $Y$ can be replaced by any formal series we see that these two identities are special cases of the expansion

$$
\mathcal{T}_{E}=\sum_{\mu} s_{\mu}[E] s_{\mu}^{\perp}
$$

The particular instances that will play a role here are obtained by setting $E= \pm 1$. These simply reduce to

$$
\mathcal{T}_{-1}=\sum_{k \geq 0}(-1)^{k} e_{k}^{\perp} \quad \text { and } \quad \mathcal{T}_{1}=\sum_{k \geq 0} h_{k}^{\perp}
$$

An interesting generalization of 1.8 is the following

Proposition 1.2 For any partition $\mu=\left(\mu_{1}, \mu_{2} \ldots, \mu_{k}\right)$ we have

$$
s_{\mu}[X-1]=\operatorname{det}\left[\begin{array}{ccccc}
1 & 1 & 1 & \cdots & 1 \\
h_{\mu_{1}-1} & h_{\mu_{1}} & h_{\mu_{1}+1} & \cdots & h_{\mu_{1}+k-1} \\
h_{\mu_{2}-2} & h_{\mu_{2}-1} & h_{\mu_{2}} & \cdots & h_{\mu_{2}+k-2} \\
\vdots & \vdots & \vdots & \ddots & \vdots \\
h_{\mu_{k}-k+1} & h_{\mu_{k}-k+2} & h_{\mu_{k}-k+3} & \cdots & h_{\mu_{k}}
\end{array}\right]
$$

with the usual convention that $h_{m}=0$ when $m<0$.

Proof. It is sufficient to illustrate the argument in a special case. So let $\mu=(4,3,1)$. In this case the Jacobi-Trudi identity gives

$$
s_{\mu}[X]=\operatorname{det}\left[\begin{array}{ccc}
h_{4} & h_{5} & h_{6} \\
h_{2} & h_{3} & h_{4} \\
0 & 1 & h_{1}
\end{array}\right]
$$


making the substitution $X \rightarrow X-1$ and using 1.8 , we get

$$
s_{\mu}[X-1]=\operatorname{det}\left[\begin{array}{ccc}
h_{4}-h_{3} & h_{5}-h_{4} & h_{6}-h_{5} \\
h_{2}-h_{1} & h_{3}-h_{2} & h_{4}-h_{3} \\
0 & 1 & h_{1}-1
\end{array}\right]=\operatorname{det}\left[\begin{array}{cccc}
1 & 1 & 1 & 1 \\
h_{3} & h_{4} & h_{5} & h_{6} \\
h_{1} & h_{2} & h_{3} & h_{4} \\
0 & 0 & 1 & h_{1}
\end{array}\right]
$$

since the first determinant is obtained from the second by subtracting from each column the preceeding column giving

$$
\operatorname{det}\left[\begin{array}{cccc}
1 & 0 & 0 & 0 \\
h_{3} & h_{4}-h_{3} & h_{5}-h_{4} & h_{6}-h_{5} \\
h_{1} & h_{2}-h_{1} & h_{3}-h_{2} & h_{4}-h_{3} \\
0 & 0 & 1 & h_{1}-1
\end{array}\right]
$$

The general case of equation 1.12 can clearly be established in a similar manner.

\section{Proofs of the umbral and recursive formulas}

After this foray into plethystic magic we are ready to play with character polynomials. Our point of departure, as in Macdonald ( $[\mathrm{Ma}]$ ), is the Frobenius formula, equation I.1, in the variables $x_{0}, x_{1}, \ldots, x_{n}$ which for $\lambda=(n-k, \mu)$ with $\mu \vdash k$ may be written in the form

$$
\chi_{\alpha}^{(n-k, \mu)}=\left.\prod_{0 \leq i<j \leq n}\left(x_{i}-x_{j}\right) p_{\alpha}\left[x_{0}+\cdots+x_{n}\right]\right|_{x_{0}^{n-k+n} x_{1}^{\mu_{1}+n-1} \cdots x_{n}^{\mu_{n}}}
$$

where for convenience we have set $\mu_{r}=0$ for all $r>\ell(\mu)$. As in [Ma] (ch. 1 ex 14), we note that the homogeneity in $x_{0}, x_{1}, \ldots, x_{n}$ of the polynomial in 2.1 allows us to set $x_{0}=1$ and reduce this identity to

$$
\begin{aligned}
\chi_{\alpha}^{(n-k, \mu)} & =\left.\prod_{i=1}^{n}\left(1-x_{i}\right) \prod_{1 \leq i<j \leq n}\left(x_{i}-x_{j}\right) p_{\alpha}\left[1+X_{n}\right]\right|_{x_{1}^{\mu_{1}+n-1} \cdots x_{n}^{\mu_{n}}} \\
& =\left.\prod_{i=1}^{n}\left(1-x_{i}\right) \mathcal{T}_{1} p_{\alpha}\right|_{s_{\mu}}=\left\langle\prod_{i=1}^{n}\left(1-x_{i}\right) \mathcal{T}_{1} p_{\alpha}, s_{\mu}\right\rangle
\end{aligned}
$$

where " $\langle$,$\rangle " denotes the Hall scalar product. Since \prod_{i=1}^{n}\left(1-x_{i}\right)=\sum_{r=0}^{n}(-1)^{r} e_{r}\left[X_{n}\right]$ we may rewrite 2.2 as

$$
\chi_{\alpha}^{(n-k, \mu)}=\left\langle\mathcal{T}_{1} p_{\alpha}, \sum_{r=0}^{n}(-1)^{r} e_{r}^{\perp} s_{\mu}\right\rangle
$$


Using the first identity in 1.11, this in turn can be rewritten as

$$
\chi_{\alpha}^{(n-k, \mu)}=\left\langle\mathcal{T}_{1} p_{\alpha}, \mathcal{T}_{-1} s_{\mu}\right\rangle .
$$

Up to this point, albeit with some slight difference of notation, we have followed Macdonald almost verbatim. To obtain our umbral formula we only need to diverge slightly from Macdonald's path. To begin we use the expansion

$$
\mathcal{T}_{-1} s_{\mu}=\sum_{\substack{\beta \vdash k \\ \beta=1^{b_{1}} 2^{b_{2} \ldots k^{b_{k}}}}} \frac{\chi_{\beta}^{\mu}}{z_{\beta}} \prod_{i=1}^{k}\left(p_{i}-1\right)^{b_{i}}
$$

and the second identity in 1.11 to rewrite 2.3 as

$$
\chi_{\alpha}^{(n-k, \mu)}=\sum_{\substack{\beta \vdash k \\ \beta=1^{b_{1}} 2^{b_{2} \ldots k^{b_{k}}}}} \frac{\chi_{\beta}^{\mu}}{z_{\beta}}\left\langle p_{\alpha}, \sum_{m=0}^{n} h_{m} \prod_{i=1}^{k}\left(p_{i}-1\right)^{b_{i}}\right\rangle .
$$

Since the operator adjoint to multiplication by $p_{i}$ is simply $i \partial_{p_{i}}$, we derive that

$$
\left\langle p_{\alpha}, \sum_{m=0}^{n} h_{m} \prod_{i=1}^{k}\left(p_{i}-1\right)^{b_{i}}\right\rangle=\left\langle\prod_{i=1}^{k}\left(i \partial p_{i}-1\right)^{b_{i}} p_{\alpha}, \sum_{m=0}^{n} h_{m}\right\rangle .
$$

Now note that for any integral vector $t=\left(t_{1}, t_{2}, \ldots, t_{n}\right)$ we have

$$
\left\langle\prod_{i=1}^{n} \partial_{p_{i}}^{t_{i}} p_{\alpha}, \sum_{m=0}^{n} h_{m}\right\rangle=\prod_{i=1}^{n}\left(a_{i}\right)_{t_{i}}\left\langle\prod_{i=1}^{n} p_{i}^{a_{i}-t_{i}}, \sum_{m=0}^{n} h_{m}\right\rangle
$$

and the identity

$$
\left\langle p_{\alpha}, h_{m}\right\rangle= \begin{cases}1 & \text { if } \alpha \vdash m \\ 0 & \text { otherwise. }\end{cases}
$$

gives

$$
\left\langle\prod_{i=1}^{n} \partial_{p_{i}}^{t_{i}} p_{\alpha}, \sum_{m=0}^{n} h_{m}\right\rangle=\prod_{i=1}^{n}\left(a_{i}\right)_{t_{i}}=\left.\downarrow \prod_{i=1}^{n} x_{i}^{t_{i}}\right|_{x_{i}=a_{i}},
$$

Expanding the first product in the right hand side of 2.5 we obtain

$$
\left\langle p_{\alpha}, \sum_{m=0}^{n} h_{m} \prod_{i=1}^{k}\left(p_{i}-1\right)^{b_{i}}\right\rangle=\left.\downarrow \prod_{i=1}^{k}\left(i x_{i}-1\right)^{b_{i}}\right|_{x_{i}=a_{i}} .
$$


In other words, equation 2.4 yields that if we set

$$
q_{\mu}\left(x_{1}, x_{2}, \ldots x_{k}\right)=\sum_{\substack{\beta \vdash k \\ \beta=1^{b_{1}} 2^{b_{2} \cdots k^{b}}}} \frac{\chi_{\beta}^{\mu}}{z_{\beta}} \downarrow \prod_{i=1}^{k}\left(i x_{i}-1\right)^{b_{i}}
$$

then $\chi_{\alpha}^{(n-k, \mu)}=\left.q_{\mu}\left(x_{1}, x_{2}, \ldots x_{k}\right)\right|_{x_{i}=a_{i}}$. This proves that the polynomial defined by equation I.5 satisfies equation I.2 as desired. Remarkably, formula 2.3, which is only a few steps away from Frobenius' formula, contains more information about Schur functions that one may suspect. Indeed using the identities in 1.11 we may rewrite equation 2.3 again as

$$
\chi_{\alpha}^{(n-k, \mu)}=\sum_{m=0}^{n} \sum_{r=0}^{n}(-1)^{r}\left\langle h_{m}^{\perp} p_{\alpha}, e_{r}^{\perp} s_{\mu}\right\rangle=\sum_{m=0}^{n} \sum_{r=0}^{n}(-1)^{r}\left\langle p_{\alpha}, h_{m} e_{r}^{\perp} s_{\mu}\right\rangle .
$$

Now note that, since the factor $p_{\alpha}$ is of degree $n$ then the scalar product $\left\langle p_{\alpha}, h_{m} e_{r}^{\perp} s_{\mu}\right\rangle$ fails to vanish only when the term $h_{m} e_{r}^{\perp} s_{\mu}$ is also of degree $n$. Since $s_{\mu}$ is of degree $k$, this can only happen when $m=n-k+r$. This simple observation yields that equation 2.7 is none other than

$$
\chi_{\alpha}^{(n-k, \mu)}=\sum_{r=0}^{k}(-1)^{r}\left\langle p_{\alpha}, h_{n-k+r} e_{r}^{\perp} s_{\mu}\right\rangle .
$$

Using again equation I.1, we can give this identity yet one more suggestive form namely

$$
\left\langle p_{\alpha}, s_{(n-k, \mu)}\right\rangle=\left\langle p_{\alpha}, \sum_{r=0}^{k}(-1)^{r} h_{n-k+r} e_{r}^{\perp} s_{\mu}\right\rangle .
$$

Since this is true for all power basis elements it follows that we must have $s_{(n-k, \mu)}=\sum_{r=0}^{k}(-1)^{r} h_{n-k+r} e_{r}^{\perp} s_{\mu}$ or better

$$
s_{(n-k, \mu)}=\mathbf{H}_{n-k} s_{\mu}
$$

where for a given integer $a \geq 0$ we set

$$
\mathbf{H}_{a}=\sum_{r \geq 0}(-1)^{r} h_{a+r} e_{r}^{\perp}
$$


Thus it follows, by recursive applications of equation 2.8 , that for any partition $\lambda=$ $\left(\lambda_{1}, \lambda_{2}, \ldots, \lambda_{k}\right)$ we have

$$
s_{\left(\lambda_{1}, \lambda_{2}, \ldots, \lambda_{k}\right)}=\mathbf{H}_{\lambda_{1}} \mathbf{H}_{\lambda_{2}} \cdots \mathbf{H}_{\lambda_{k}} \mathbf{1},
$$

which is the well known "Rodrigues" formula for Schur functions (see [WW]). What we find surprising is that equation 2.9 as well as 2.10 could be such short distance away from the original Frobenius formula. Even more surprising would be if the present derivation of 2.9 did not previously appear in the extensive literature on Schur functions.

Remark 2.1. It is easy to see that the action of $\mathbf{H}_{a}$ on any symmetric polynomial $A(X)$ may be given the plethystic form

$$
\mathbf{H}_{a} A(X)=\left.\Omega[z X] A[X-1 / z]\right|_{z^{a}}
$$

Under this form, the operator $\sum_{a} \mathbf{H}_{a} z^{a}$ has acquired the name of "vertex operator", (see $[\mathrm{CT}]$ ). Using this notation, it is not difficult to derive the commutativity relation $\mathbf{H}_{a} \mathbf{H}_{b}=-\mathbf{H}_{b-1} \mathbf{H}_{a+1}$ from which it follows that equation 2.10 remains valid even when $\lambda_{1}, \lambda_{2}, \ldots, \lambda_{k}$ are not in weakly decreasing order.

Remark 2.2. The operation that gave us the character polynomial can be extended to a linear map that sends symmetric polynomials onto polynomials in the infinite alphabet $X=\left\{x_{1}, x_{2}, x_{3}, \ldots\right\}$. More precisely for each symmetric function $A$ of degree $k$ we define the map $\mathbf{q}: \Lambda \rightarrow \mathbf{Q}[X]$ by setting

$$
\mathbf{q}(A)=\mathbf{q}_{A}\left[x_{1}, x_{2}, \ldots, x_{k}\right]=\left.\downarrow Q_{A}\left(p_{1}, p_{2}, \ldots, p_{k}\right)\right|_{p_{i} \rightarrow i x_{i}-1}
$$

where $Q_{A}$ gives the power sum expansion of $A$. Clearly, using this notation we can also write $q_{\mu}=\mathbf{q}\left(s_{\mu}\right)$ We shall see in the next section that the map $A \rightarrow \mathbf{q}(A)$ has truly remarkable properties. But for the moment we will use it only as a convenient notation.

\section{Proof of Theorem I.1}

Note that since multiplication of a Schur function by the power symmetric function $p_{i}$ corresponds to the addition of a border strip of size $i$ to its index, it follows that applying $i \partial_{p_{i}}=p_{i}^{\perp}$ to a Schur function corresponds to the removal of such a strip. In summary, we see that the combinatorial statement of theorem I.1 is simply equivalent to the following polynomial identity

$$
q_{\mu}\left(x_{1}, x_{2}, \ldots, x_{k}\right)=q_{\mu}\left(x_{1}\right)+\left.\sum_{i=2}^{k} \sum_{s=1}^{k / i}\left(\begin{array}{c}
x_{i} \\
s
\end{array}\right) \mathbf{q}\left(\left(i \partial_{p_{i}}\right)^{s} s_{\mu}\right)\right|_{x_{i}=x_{i+1}=\cdots=x_{k}=0} .
$$

the electronic Journal of Combinatorics 16(2) (2009), \#R19 
Now, recalling that $z_{\theta}=1^{t_{1}} 2^{t_{2}} \cdots k^{t_{k}} t_{1} ! t_{2} ! \cdots t_{k}$ !, we have

$$
\begin{aligned}
\mathbf{q}\left(\left(i \partial_{p_{i}}\right)^{s} s_{\mu}\right) & =\left.i^{s} \sum_{\theta \vdash k} \frac{\chi_{\theta}^{\mu}}{z_{\theta}} \downarrow \partial_{p_{i}}^{s} p_{1}^{t_{1}} p_{2}^{t_{2}} \cdots p_{k}^{t_{k}}\right|_{p_{j} \rightarrow\left(j x_{j}-1\right)} \\
& =\left.i^{s} \sum_{\theta \vdash k} \frac{\chi_{\theta}^{\mu}}{z_{\theta}}\left(t_{i}\right)_{s} \downarrow p_{1}^{t_{1}} \cdots p_{i}^{t_{i}-s} \cdots p_{k}^{t_{k}}\right|_{p_{j} \rightarrow\left(j x_{j}-1\right)} \\
& =i^{s} \sum_{\theta \vdash k} \frac{\chi_{\theta}^{\mu}}{z_{\theta}}\left(t_{i}\right)_{s} \prod_{j=1}^{i-1} \downarrow\left(j x_{j}-1\right)^{t_{j}} \times \downarrow\left(i x_{i}-1\right)^{t_{i}-s} \times \prod_{j=i+1}^{k} \downarrow\left(j x_{j}-1\right)^{t_{j}}
\end{aligned}
$$

Thus

$$
\left.\mathbf{q}\left(\left(i \partial_{p_{i}}\right)^{s} s_{\mu}\right)\right|_{x_{i}=x_{i+1}=\cdots=x_{k}=0}=i^{s} \sum_{\theta \vdash k} \frac{\chi_{\theta}^{\mu}}{z_{\theta}}\left(t_{i}\right)_{s} \prod_{j=1}^{i-1} \downarrow\left(j x_{j}-1\right)^{t_{j}} \times(-1)^{t_{i}-s} \prod_{j=i+1}^{k}(-1)^{t_{j}}
$$

and using this we get

$$
\begin{aligned}
\sum_{s=1}^{k / i}\left(\begin{array}{c}
x_{i} \\
s
\end{array}\right) & \left.\mathbf{q}\left(\left(i \partial_{p_{i}}\right)^{s} s_{\mu}\right)\right|_{x_{i}=\cdots=x_{k}=0} \\
& =\sum_{\theta \vdash k} \frac{\chi_{\theta}^{\mu}}{z_{\theta}} \sum_{s=1}^{k / i}\left(\begin{array}{c}
x_{i} \\
s
\end{array}\right) i^{s}\left(t_{i}\right)_{s}(-1)^{t_{i}-s} \prod_{j=1}^{i-1} \downarrow\left(j x_{j}-1\right)^{t_{j}} \times(-1)^{\sum_{j=i+1}^{k} t_{j}}
\end{aligned}
$$

$\left(s \leq t_{i} \rightarrow s i \leq k\right)$

$$
\begin{aligned}
& =\sum_{\theta \vdash k} \frac{\chi_{\theta}^{\mu}}{z_{\theta}} \sum_{s=1}^{t_{i}}\left(\begin{array}{c}
t_{i} \\
s
\end{array}\right) i^{s}\left(x_{i}\right)_{s}(-1)^{t_{i}-s} \prod_{j=1}^{i-1} \downarrow\left(j x_{j}-1\right)^{t_{j}} \times(-1)^{\sum_{j=i+1}^{k} t_{j}} \\
& =\sum_{\theta \vdash k} \frac{\chi_{\theta}^{\mu}}{z_{\theta}} \downarrow\left(\left(i x_{i}-1\right)^{t_{i}}-(-1)^{t_{i}}\right) \prod_{j=1}^{i-1} \downarrow\left(j x_{j}-1\right)^{t_{j}} \times(-1)^{\sum_{j=i+1}^{k} t_{j}} \\
& =\sum_{\theta \vdash k} \frac{\chi_{\theta}^{\mu}}{z_{\theta}} \prod_{j=1}^{i} \downarrow\left(j x_{j}-1\right)^{t_{j}}(-1)^{\sum_{j=i+1}^{k} t_{j}}-\sum_{\theta \vdash k} \frac{\chi_{\theta}^{\mu}}{z_{\theta}} \prod_{j=1}^{i-1} \downarrow\left(j x_{j}-1\right)^{t_{j}}(-1)^{\sum_{j=i}^{k} t_{j}} \\
& =\left.q_{\mu}\left(x_{1}, x_{2}, \ldots, x_{k}\right)\right|_{x_{i+1}=\cdots=x_{k}=0}-\left.q_{\mu}\left(x_{1}, x_{2}, \ldots, x_{k}\right)\right|_{x_{i}=\cdots=x_{k}=0}
\end{aligned}
$$

and equation 2.12 becomes

$$
q_{\mu}\left(x_{1}, x_{2}, \ldots, x_{k}\right)=q_{\mu}\left(x_{1}\right)+\sum_{i=2}^{k}\left(q_{\mu}\left(x_{1}, \ldots, x_{i}\right)-q_{\mu}\left(x_{1}, \cdots, x_{i-1}\right)\right)
$$

THE ELECTRONiC JOURNAL OF COMBINATORICS 16(2) (2009), \#R19 
which is obviously true.

\section{Basics on the Kronecker product}

Let $C_{\alpha}$ denote the formal sum of the permutations of $S_{n}$ with cycle type $\alpha$ and let $\mathcal{C}\left(S_{n}\right)$ denote the center of the group algebra of $S_{n}$. The map $\mathbf{F}_{n}: \mathcal{C}\left(S_{n}\right) \rightarrow \Lambda=n$ defined by setting

$$
\mathbf{F}_{n}\left(C_{\alpha}\right)=p_{\alpha} / z_{\alpha},
$$

usually called the "Frobenius map", was the tool used by Frobenius to identify the characters of $S_{n}$. In fact this map is an isometry of $\mathcal{C}\left(S_{n}\right)$ onto $\Lambda^{=n}$ each endowed with its natural scalar product. The orthonormality of the Schur basis together with its integrality with respect to the homogeneous basis $\left\{h_{\alpha}\right\}_{\alpha \vdash n}$ yielded Frobenius the fundamental relation

$$
s_{\lambda}=\mathbf{F}_{n}\left(\chi^{\lambda}\right)=\sum_{\alpha \vdash n} \chi_{\alpha}^{\lambda} p_{\alpha} / z_{\alpha}
$$

which he inverted to

$$
p_{\alpha}=\sum_{\lambda \vdash n} \chi_{\alpha}^{\lambda} s_{\lambda}
$$

and then equation I.1 followed from the bideterminantal formula for Schur functions.

The Kronecker product in $\Lambda^{=n}$ is defined by setting for $P, Q \in \Lambda^{=n}$

$$
P * Q=\mathbf{F}_{n}\left[\mathbf{F}_{n}^{-1}(P) \times \mathbf{F}_{n}^{-1}(Q)\right]
$$

where the symbol " $\times$ " here represents the pointwise product in $\mathcal{C}\left(S_{n}\right)$. In particular from equation 3.2 it follows that

$$
s_{\mu} * s_{\nu}=\sum_{\alpha \vdash n} \chi_{\alpha}^{\mu} \chi_{\alpha}^{\nu} p_{\alpha} / z_{\alpha} \quad(\text { for all } \mu, \nu \vdash n) .
$$

The orthonormality of the Schur functions then yields the expansion

$$
s_{\mu} * s_{\nu}=\sum_{\lambda \vdash n} c_{\lambda, \mu, \nu} s_{\lambda}
$$

with

$$
c_{\lambda, \mu, \nu}=\left\langle s_{\mu} * s_{\nu}, s_{\lambda}\right\rangle=\sum_{\alpha \vdash n} \chi_{\alpha}^{\lambda} \chi_{\alpha}^{\mu} \chi_{\alpha}^{\nu} / z_{\alpha}
$$

where the last equality is obtained by combining equation 3.5 with 3.2 and the relation

$$
\left\langle p_{\alpha}, p_{\beta}\right\rangle= \begin{cases}z_{\alpha} & \text { if } \alpha=\beta, \\ 0 & \text { if } \alpha \neq \beta .\end{cases}
$$


The $c_{\lambda, \mu, \nu}$ go by the name of "Kronecker coefficients" and they may be interpreted as the multiplicity of the irreducible representation of $S_{n}$ indexed by $\lambda$ in the tensor product of the irreducible representations indexed by $\mu$ and $\nu$. A fundamental open problem is to give a combinatorial interpretation to these integers akin to the Littlewood Richardson rule for the coefficients $g_{\lambda, \mu, \nu}$ occuring in the expansion

$$
s_{\mu} s_{\nu}=\sum_{\lambda} g_{\lambda, \mu, \nu} s_{\lambda}
$$

So far, explicit expressions for the coefficients $c_{\lambda, \mu, \nu}$ have been given only for very special choices of $\lambda, \mu, \nu$. The relation in equation 3.6 enables us to attack the Kronecker coefficient problem by symmetric function methods. In this writing we will focus on the identities that reduce the computation of the Kronecker products of symmetric functions to ordinary products and thereby ultimately express the $c_{\lambda, \mu, \nu}$ in terms of the $g_{\lambda, \mu, \nu}$. To this end it is convenient to extend the Kronecker product to all of $\Lambda$ by setting

$$
P * Q=0 \quad \text { for all pairs } P, Q \text { with } P \in \Lambda^{=r}, Q \in \Lambda^{=s} \text { and } r \neq s
$$

This given, the following basic identities are immediate consequences of the definition of Kronecker products given in equation 3.4.

\section{Proposition 3.1}

1) $p_{\alpha} * p_{\beta}=\chi(\alpha=\beta) z_{\alpha} p_{\alpha}, \quad$ for all pairs of partitions $\alpha, \beta$

2) $\quad p_{\alpha} * s_{\lambda}=\chi_{\alpha}^{\lambda} p_{\alpha}, \quad$ for all pairs of partitions $\alpha, \lambda \vdash n$

3) $\Omega * f=h_{n} * f=f, \quad$ for all $f \in \Lambda^{=n}$

where $\chi(\alpha=\beta)$ is the Kronecker delta function.

Proof. Since conjugacy classes are disjoint, it follows that (" $\times$ " denoting pointwise product) we have

$$
C_{\alpha} \times C_{\beta}= \begin{cases}C_{\alpha} & \text { if } \alpha=\beta \\ 0 & \text { if } \alpha \neq \beta\end{cases}
$$

Thus 1) follows from equations 3.1 and 3.4 when $\alpha$ and $\beta$ are partitions of the same number and from equation 3.9 when they are not. This given, 2) follows by linearity from equation 3.2. Finally, 3) follows from equation 3.9 together with 1) and the well known expansion

$$
h_{n}=\sum_{\alpha \vdash n} p_{\alpha} / z_{\alpha} .
$$

THE ELECTRONiC JOURnAl OF COMBINATORICS 16(2) (2009), \#R19 
The following basic identity of Littlewood ([Li]) provides an algorithm for the computation of Kronecker products.

Proposition 3.2 For any homogeneous symmetric functions $f_{1}, f_{2}, \ldots, f_{k}$ of respective degrees $a_{1}, a_{2}, \ldots, a_{k}$ and any symmetric function $A$ of degree $a_{1}+a_{2}+\cdots+a_{k}$, we have

$$
\begin{aligned}
& \left(f_{1} f_{2} \cdots f_{k}\right) * A \\
& =\sum_{\alpha^{(1)} \vdash a_{1}} \sum_{\alpha^{(2)} \vdash a_{2}} \cdots \sum_{\alpha^{(k)} \vdash a_{k}}\left\langle s_{\alpha^{(1)}} s_{\alpha^{(2)}} \cdots s_{\alpha^{(k)}}, A\right\rangle\left(f_{1} * s_{\alpha^{(1)}}\right)\left(f_{2} * s_{\alpha^{(2)}}\right) \cdots\left(f_{k} * s_{\alpha^{(k)}}\right),
\end{aligned}
$$

In particular we derive that

$$
\begin{aligned}
\left(h_{\mu_{1}} h_{\mu_{2}} \cdots h_{\mu_{k}}\right) * A & =\sum_{\alpha^{(1) \vdash \mu_{1}}} \sum_{\alpha^{(2)} \vdash \mu_{2}} \cdots \sum_{\alpha^{(k)} \vdash \mu_{k}}\left\langle s_{\alpha^{(1)}} s_{\alpha^{(2)}} \cdots s_{\alpha^{(k)}}, A\right\rangle s_{\alpha^{(1)}} s_{\alpha^{(2)}} \cdots s_{\alpha^{(k)}} \\
& =\sum_{\alpha^{(2) \vdash \mu_{2}}} \cdots \sum_{\alpha^{(k) \vdash \mu_{k}}} s_{\alpha^{(2)}} \cdots s_{\alpha^{(k)}} s_{\alpha^{(2)}}^{\perp} \cdots s_{\alpha^{(k)}}^{\perp} A
\end{aligned}
$$

where the $s_{\alpha^{(i)}}^{\perp}$ and $s_{\alpha^{(i)}}$ are seen as skewing and multiplication operators successively applied on $A$.

Proof. We need only prove equation 3.12 for $f_{i}=p_{\beta^{(i)}}$ with $\beta^{(i)} \vdash a_{i}$ and $A=s_{\lambda}$ with $\lambda \vdash a_{1}+\cdots+a_{k}$. This given, using 3.102 ), the left hand side of equation 3.12 becomes

$$
\begin{aligned}
L H S=p_{\beta^{(1)}} p_{\beta^{(2)}} \cdots p_{\beta^{(k)}} * s_{\lambda} & =\chi_{\beta^{(1)} \vee \beta^{(2)} \ldots \vee \beta^{(k)}}^{\lambda} p_{\beta^{(1)} \vee \beta^{(2)} \ldots \vee \beta^{(k)}} \\
& =\left\langle p_{\beta^{(1)} \vee \beta^{(2)} \ldots \vee \beta^{(k)}}, s_{\lambda}\right\rangle p_{\beta^{(1)} \vee \beta^{(2)} \ldots \vee \beta^{(k)}} .
\end{aligned}
$$

On the other hand, using again equation 3.102 ), the right hand side of equation 3.12 becomes

$$
R H S=\sum_{\alpha^{(1)} \vdash a_{1}} \cdots \sum_{\alpha^{(k)} \vdash a_{k}}\left\langle s_{\alpha^{(1)}} \cdots s_{\alpha^{(k)}}, s_{\lambda}\right\rangle \chi_{\beta^{(1)}}^{\alpha^{(1)}} p_{\beta^{(1)}} \cdots \chi_{\beta^{(1)}}^{\alpha^{(1)}} p_{\beta^{(1)}}
$$

and equation 3.12 in this case immediately follows from the Frobenius expansion in equation 3.3. This given, the first equality in equation 3.13 follows by setting $f_{i}=h_{\mu_{i}}$ and using equation 3.103 ). The second equality follows from the first and the fact that $\left\langle s_{\alpha^{(1)}} \cdots s_{\alpha^{(k)}}, A\right\rangle=\left\langle s_{\alpha^{(1)}}, s_{\alpha^{(2)}}^{\perp} \cdots s_{\alpha^{(k)}}^{\perp} A\right\rangle$. This completes our proof.

Remark 3.1. Murnaghan ([Mu]) noted that for any partition $\mu \vdash k$, the Kronecker multiplication operator " $s_{(n-k, \mu)} *$ " does not depend on $n$ in the sense that the value of 
the Kronecker coefficients $c_{(n-|\lambda|, \lambda),(n-|\mu|, \mu),(n-|\nu|, \nu)}$ stabilizes when $n$ exceeds a lower bound depending on $\lambda, \mu, \nu$. We can easily derive this from the identities in equations 2.3 and 3.13. Indeed from equation 2.3 it follows that we can write

$$
\begin{aligned}
s_{(n-k, \mu)} & =\sum_{\alpha \vdash n}\left\langle s_{(n-k, \mu)}, p_{\alpha}\right\rangle p_{\alpha} / z_{\alpha} \\
& =\sum_{\alpha \vdash n}\left\langle\mathcal{T}_{-1} s_{\mu}, \mathcal{T}_{1} p_{\alpha}\right\rangle p_{\alpha} / z_{\alpha} \\
\text { (Using 1.11) } & =\sum_{\alpha \vdash n} \sum_{m \geq 0}\left\langle h_{m} s_{\mu}[X-1], T_{1} p_{\alpha}\right\rangle p_{\alpha} / z_{\alpha} \\
& =\sum_{\alpha \vdash n} \sum_{r \geq 0}^{n}\left\langle h_{n-r} s_{\mu}^{(r)}[X-1], T_{1} p_{\alpha}\right\rangle p_{\alpha} / z_{\alpha}
\end{aligned}
$$

where " $s_{\mu}^{(r)}[X-1]$ " denotes the homogeneous component of degree $r$ in $s_{\mu}[X-1]$. This implies that

$$
s_{(n-k, \mu)}[X]=\sum_{r \geq 0} h_{n-r} s_{\mu}^{(r)}[X-1]
$$

Thus if the homogeneous basis expansion of $s_{\mu}[X-1]$ is

$$
s_{\mu}[X-1]=\sum_{r=0}^{k} \sum_{\alpha \vdash r} c_{\mu \alpha} h_{\alpha_{1}} h_{\alpha_{2}} \cdots h_{\alpha_{r}} \quad \text { (allowing some } \alpha_{i} \text { to vanish) }
$$

then equation 3.14 becomes

$$
s_{(n-k, \mu)}=\sum_{r=0}^{k} \sum_{\alpha \vdash r} c_{\mu \alpha} h_{n-r} h_{\alpha_{1}} h_{\alpha_{2}} \cdots h_{\alpha_{r}}
$$

and the equality in equation 3.13 gives $s_{(n-k, \mu)} * A=\mathbf{U}_{\mu}(A)$ for all $A$ of degree $n$ with

$$
\mathbf{U}_{\mu}=\sum_{r=0}^{k} \sum_{\alpha \vdash r} c_{\mu \alpha} \sum_{\gamma^{(1)} \vdash \alpha_{1}} \sum_{\gamma^{(2)} \vdash \alpha_{2}} \cdots \sum_{\gamma^{(r)} \vdash \alpha_{r}} s_{\gamma^{(1)}} s_{\gamma^{(2)}} \cdots s_{\gamma^{(r)}} s_{\gamma^{(1)}}^{\perp} s_{\gamma^{(2)}}^{\perp} \cdots s_{\gamma^{(r)}}^{\perp}
$$

This explicit version of Murnaghan's assertion was given in [GC]. Proposition 3.1 has a beautiful corollary, again due to Littlewood ([Li]), that may be stated as follows.

Theorem 3.1 For any homogeneous basis element $h_{\alpha}=h_{\alpha_{1}} h_{\alpha_{2}} \cdots h_{\alpha_{k}}$ and any skew diagram $D$ with $n=\alpha_{1}+\alpha_{2}+\cdots+\alpha_{k}$ cells we have

$$
h_{\alpha} * s_{D}=\sum_{\substack{D_{1}+D_{2}+\cdots+D_{k}=D \\\left|D_{i}\right|=\alpha_{i}}} s_{D_{1}} s_{D_{2}} \cdots s_{D_{k}} .
$$

THE ELECTRONiC JOURNAL OF COMBINATORICS 16(2) (2009), \#R19 
where the sum is over all decompositions of $D$ as a disjoint union of $k$ skew diagrams $D_{i}$ containing $\alpha_{i}$ cells.

Proof. We prove first the case $k=2$. So let $D=\gamma / \delta$ with $\gamma \vdash a+b+c$ and $\delta \vdash c$. Note that from equation 3.13 for $k=2$ with $\mu_{1}=a, \mu_{2}=b$ and $A=s_{\gamma / \delta}$ we get

$$
\begin{aligned}
h_{a} h_{b} * s_{\gamma / \delta} & =\sum_{\mu \vdash a} \sum_{\nu \vdash b}\left\langle s_{\gamma / \delta}, s_{\mu} s_{\nu}\right\rangle s_{\mu} s_{\nu} \\
& =\sum_{\mu \vdash a} \sum_{\nu \vdash b}\left\langle s_{\gamma / \nu}, s_{\delta} s_{\mu}\right\rangle s_{\mu} s_{\nu}
\end{aligned}
$$

Substituting in this the expansion

$$
s_{\gamma / \nu}=\sum_{\lambda \vdash a+c}\left\langle s_{\gamma / \nu}, s_{\lambda}\right\rangle s_{\lambda}=\sum_{\lambda \vdash a+c}\left\langle s_{\gamma}, s_{\nu} s_{\lambda}\right\rangle s_{\lambda}
$$

we get

$$
\begin{aligned}
h_{a} h_{b} * s_{\gamma / \delta} & =\sum_{\mu \vdash a} \sum_{\nu \vdash b} \sum_{\lambda \vdash a+c}\left\langle s_{\gamma}, s_{\nu} s_{\lambda}\right\rangle\left\langle s_{\lambda}, s_{\delta} s_{\mu}\right\rangle s_{\mu} s_{\nu} \\
& =\sum_{\mu \vdash a} \sum_{\nu \vdash b} \sum_{\lambda \vdash a+c}\left\langle s_{\gamma / \lambda}, s_{\nu}\right\rangle\left\langle s_{\lambda / \delta}, s_{\mu}\right\rangle s_{\mu} s_{\nu}=\sum_{\lambda \vdash a+c} s_{\lambda / \delta} s_{\gamma / \lambda}
\end{aligned}
$$

and this is simply another way of writing equation 3.16 for $k=2$. We may thus proceed by induction on $k$. So assume equation 3.16 true for some $k \geq 2$. We then proceed as before and let $D=\gamma / \delta$ with $\gamma \vdash a+b+c$ and $\delta \vdash c$, and get from 3.12 for $k=2, f_{1}=h_{a}$ and $f_{2}=h_{\alpha}=h_{\alpha_{1}} h_{\alpha_{2}} \cdots h_{\alpha_{k}}$

$$
\begin{aligned}
h_{a} h_{\alpha} * s_{D} & =\sum_{\mu \vdash a} \sum_{\nu \vdash b}\left\langle s_{\gamma / \delta}, s_{\mu} s_{\nu}\right\rangle s_{\mu} h_{\alpha} * s_{\nu} \\
& =\sum_{\mu \vdash a} \sum_{\nu \vdash b}\left\langle s_{\gamma}, s_{\delta} s_{\mu} s_{\nu}\right\rangle s_{\mu} h_{\alpha} * s_{\nu} \\
& =\sum_{\mu \vdash a} \sum_{\nu \vdash b}\left\langle s_{\gamma / \nu}, s_{\delta} s_{\mu}\right\rangle s_{\mu} h_{\alpha} * s_{\nu} \\
& =\sum_{\mu \vdash a} \sum_{\nu \vdash b} \sum_{\lambda \vdash a+c}\left\langle s_{\gamma / \nu}, s_{\lambda}\right\rangle\left\langle s_{\lambda}, s_{\delta} s_{\mu}\right\rangle s_{\mu} h_{\alpha} * s_{\nu} \\
& =\sum_{\mu \vdash a} \sum_{\nu \vdash b} \sum_{\lambda \vdash a+c}\left\langle s_{\gamma / \lambda}, s_{\nu}\right\rangle\left\langle s_{\lambda / \delta}, s_{\mu}\right\rangle s_{\mu} h_{\alpha} * s_{\nu} \\
& =\sum_{\lambda \vdash a+c} s_{\lambda / \delta} h_{\alpha} * s_{\gamma / \lambda}
\end{aligned}
$$

THE ELECTRONiC JOURNAL OF COMBINATORICS 16(2) (2009), \#R19 
So the induction hypothesis for $\alpha=\left(\alpha_{1}, \alpha_{2}, \ldots, \alpha_{k}\right)$ gives

$$
h_{a} h_{\alpha} * s_{D}=\sum_{\lambda \vdash a+c} s_{\lambda / \delta} \sum_{\substack{D_{1}+D_{2}+\cdots+D_{k}=\gamma / \lambda \\\left|D_{i}\right|=\alpha_{i}}} s_{D_{1}} s_{D_{2}} \cdots s_{D_{k}}
$$

this proves equation 3.16 for $k+1$ and completes the induction.

It is worthwhile to close this section with an example illustrating a use of these identities in the computation of Kronecker products. Let us compute the operator $\mathbf{U}_{21}$ defined in equation 3.15. To this end note first that equation 1.12 gives

$$
s_{21}[X-1]=\operatorname{det}\left[\begin{array}{ccc}
1 & 1 & 1 \\
h_{1} & h_{2} & h_{3} \\
0 & 1 & h_{1}
\end{array}\right]=h_{2} h_{1}-h_{3}-h_{1} h_{1}+h_{1}
$$

and equation 3.15 gives

$$
U_{(2,1)}=s_{2} s_{1} s_{2}^{\perp} s_{1}^{\perp}+s_{11} s_{1} s_{11}^{\perp} s_{1}^{\perp}-\left(s_{3} s_{3}^{\perp}+s_{(2,1)} s_{(2,1)}^{\perp}+s_{1^{3}} s_{1^{3}}^{\perp}\right)-s_{1} s_{1} s_{1}^{\perp} s_{1}^{\perp}+s_{1} s_{1}^{\perp}
$$

Applying $U_{(2,1)}$ to $s_{n-3,3}$ we obtain one of the many identities given in $([\mathrm{Mu}])$.

$$
\begin{aligned}
s_{n-3,2,1} * s_{n-3,3}= & s_{n-1,1}+2 s_{n-2,2}+2 s_{n-2,1^{2}}+2 s_{n-3,3}+5 s_{n-3,2,1}+2 s_{n-3,1^{3}} \\
& +2 s_{n-4,4}+5 s_{n-4,3,1}+3 s_{n-4,2^{2}}+4 s_{n-4,2,1^{2}}+s_{n-4,1^{4}}+s_{n-5,5} \\
& +3 s_{n-5,4,1}+3 s_{n-5,3,2}+3 s_{n-5,3,1^{2}}+2 s_{n-5,2^{2} 1}+s_{n-5,2,1^{3}} \\
& +s_{n-6,3,2,1}+s_{n-6,5,1}+s_{n-6,4,2}+s_{n-6,4,1,1}
\end{aligned}
$$

\section{Character polynomials and the Kronecker product.}

We start with the definition of a degree in $Q\left[x_{1}, x_{2}, \ldots\right]$, different from the usual degree and we call it the "partition degree":

$$
\operatorname{pdeg}\left(x_{1}^{a_{1}} x_{2}^{a_{2}} \cdots x_{k}^{a_{k}}\right)=\sum_{i=1}^{k} i a_{i}
$$

We thus obtain a direct sum decomposition

$$
Q\left[x_{1}, x_{2}, \ldots\right]=\bigoplus_{k \geq 0} \mathcal{H}_{k}\left(x_{1}, x_{2}, \ldots, x_{k}\right)
$$


where $\mathcal{H}_{k}\left(x_{1}, x_{2}, \ldots, x_{k}\right)$ is the subspace of polynomials in $Q\left[x_{1}, x_{2}, \ldots\right]$ that are homogeneous of partition degree $k$. Note that polynomials of partition degree $\leq k$ can only contain the variables $x_{1}, x_{2}, \ldots, x_{k}$. It is also convenient to set

$$
\mathcal{H}_{\leq k}\left(x_{1}, x_{2}, \ldots, x_{k}\right)=\bigoplus_{r \geq 0}^{k} \mathcal{H}_{r}\left(x_{1}, x_{2}, \ldots, x_{k}\right)
$$

Since symmetric functions can be viewed as polynomials in $\mathbf{Q}\left[p_{1}, p_{2}, \ldots\right]$ our map $\mathbf{q}$ : $\Lambda \rightarrow \mathbf{Q}\left[x_{1}, x_{2}, \ldots\right]$ can be defined by setting (for $\alpha=1^{a_{1}} 2^{a_{2}} \cdots k^{a_{k}}$ )

$$
\mathbf{q}_{p_{\alpha}}\left(x_{1}, x_{2}, \ldots\right)=\mathbf{q}\left(p_{1}^{a_{1}} p_{2}^{a_{2}} \cdots p_{k}^{a_{k}}\right)=\downarrow \prod_{i=1}^{k}\left(i x_{i}-1\right)^{a_{i}}
$$

We should note that $\mathbf{q}$ maps the space $\Lambda \leq k$ of symmetric functions of degree $\leq k$ onto $\mathcal{H}_{\leq k}\left(x_{1}, x_{2}, \ldots, x_{k}\right)$ and since $\mathbf{q}\left(p_{\alpha}\right)=\left(\prod_{i=1}^{k} i^{a_{i}}\right) \prod_{i=1}^{k} x_{i}^{a_{i}}+\cdots$, with the omitted terms of lesser partition degree, we see that this map is degree preserving and non singular. In particular it immediately follows that the collection $\left\{q_{\mu}\right\}_{\mu}$ of character polynomials is necessarily a basis of $Q\left[x_{1}, x_{2}, \ldots\right]$. This given, we can define a scalar product $\langle\langle\rangle$,$\rangle on Q\left[x_{1}, x_{2}, \ldots\right]$ by declaring that the character polynomials form an orthonormal system. In symbols we set

$$
\left\langle\left\langle q_{\mu}, q_{\nu}\right\rangle\right\rangle= \begin{cases}1 & \text { if } \mu=\nu \\ 0 & \text { if } \mu \neq \nu\end{cases}
$$

To show that this definition is natural, we need only prove that this scalar product may be computed without using character polynomials. This is an immediate consequence of the following basic fact.

Proposition 4.1 For any polynomials $P\left(x_{1}, x_{2}, \ldots, x_{k}\right), Q\left(x_{1}, x_{2}, \ldots, x_{k}\right)$ of partition degree $\leq k$ we have

$$
\langle\langle P, Q\rangle\rangle=\sum_{\alpha \vdash n} \frac{P\left(a_{1}, a_{2}, \ldots, a_{k}\right) Q\left(a_{1}, a_{2}, \ldots, a_{k}\right)}{1^{a_{1}} 2^{a_{2}} \ldots n^{a_{n}} a_{1} ! a_{2} ! \ldots a_{n} !} \quad \forall n \geq 2 k
$$

Proof. Since the collection of character polynomials $\left\{q_{\mu}\right\}_{|\mu| \leq k}$ is known to be a basis for $\mathcal{H}_{\leq k}\left(x_{1}, x_{2}, \ldots, x_{k}\right)$ we need only check that equation 4.6 holds true for all pairs of elements of $\left\{q_{\mu}\right\}_{|\mu| \leq k}$. Now this is a simple consequence of equation I.2. In fact, both $(n-|\mu|, \mu)$ and $(n-|\nu|, \nu)$ are partitions for all $n \geq 2 k$ since the hypotheses $|\mu|,|\nu| \leq k$ 
assure that $n-|\mu| \geq \mu_{1}$ and $n-|\nu| \geq \nu_{1}$, thus equation I.2 gives $q_{\mu}\left(a_{1}, \ldots, a_{n}\right)=$ $\chi_{\alpha}^{(n-|\mu|, \mu)}$ and $q_{\nu}\left(a_{1}, \ldots, a_{n}\right)=\chi_{\alpha}^{(n-|\nu|, \nu)}$ and equation 4.6 becomes

$$
\left\langle\left\langle q_{\mu}, q_{\nu}\right\rangle\right\rangle=\sum_{\alpha \vdash n} \frac{\chi_{\alpha}^{(n-|\mu|, \mu)} \chi_{\alpha}^{(n-|\nu|, \nu)}}{z_{\alpha}}
$$

which follows from the orthonormality of the irreducible characters of $S_{n}$.

Using this result, we can in principle obtain the expansion of any polynomial $P \in \mathcal{H}_{\leq k}\left(x_{1}, x_{2}, \ldots\right)$ by the formula

$$
P\left(x_{1}, x_{2}, \ldots, x_{k}\right)=\sum_{|\alpha| \leq k}\left\langle\left\langle P, q_{\alpha}\right\rangle\right\rangle q_{\alpha}\left(x_{1}, x_{2}, \ldots, x_{k}\right)
$$

However for large $k$ we found that expansion of a polynomial $P$ in terms of character polynomials can be obtained much faster by the following algorithm:

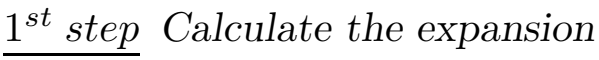

$$
P\left(x_{1}, x_{2}, \ldots, x_{k}\right)=\sum_{|\alpha| \leq k} c_{\alpha} q_{p_{\alpha}}\left(x_{1}, x_{2}, \ldots, x_{k}\right)
$$

$2^{\text {nd }}$ step Make the substitution in equation 4.7

$$
q_{p_{\alpha}}(x)=\sum_{|\mu|=\mid \alpha} \chi_{\alpha}^{\mu} q_{\mu}(x)
$$

and obtain

$$
P\left(x_{1}, x_{2}, \ldots, x_{k}\right)=\sum_{|\alpha| \leq k} c_{\alpha} \sum_{|\mu|=\mid \alpha} \chi_{\alpha}^{\mu} q_{\mu}\left(x_{1}, x_{2}, \ldots, x_{k}\right)
$$

Note that since we have

$$
q_{p_{\alpha}}\left(x_{1}, x_{2}, \ldots, x_{k}\right)=\left(\prod_{i=1}^{k} i^{a_{i}}\right) \prod_{i=1}^{k} x_{i}^{a_{i}}+\cdots
$$

with the remaining terms of lesser partition degree, we can efficiently obtain the expansion in equation 4.7 by a sequence of extractions of lexicographically leading terms as indicated by the following recursion

$$
P_{i}\left(x_{1}, x_{2}, \ldots, x_{k}\right)=P_{i-1}\left(x_{1}, x_{2}, \ldots, x_{k}\right)-\frac{\ell c\left(P_{i-1}\right)}{\prod_{i=1}^{k} i^{a_{i}}} q_{p_{\alpha}}\left(x_{1}, x_{2}, \ldots, x_{k}\right)
$$

THE ELECTRONIC JOURNAL OF COMBINATORICS 16(2) (2009), \#R19 
where we set $P(x)=P_{0}(x), \prod_{i=1}^{k} x_{i}^{a_{i}}$ is the leading monomial of $P_{i-1}$ and $\ell c\left(P_{i-1}\right)$ its leading coefficient.

Remark 4.1. Note that in computing equation 4.7 with the recursion in equation 4.10 and the substitution in equation 4.8 , we never get out of the subspace $\mathbb{Q}\left[x_{1}, x_{2}, \ldots, x_{k}\right]$. However, this is all that we can say in general. Indeed, if $P$ has partition degree $k$, no matter how restricted is the set of variables that occur in $P$, the substitution in equation 4.8 may introduce other variables in the set $\left\{x_{1}, x_{2}, \ldots, x_{k}\right\}$ in the final result. The implications of this simple observation will soon be apparent.

Murnaghan $([\mathrm{Mu}])$ noted that the Kronecker coefficients

$$
c_{(n-|\lambda|, \lambda),(n-|\mu|, \mu),(n-|\nu|, \nu)}=\sum_{\rho \vdash n} \chi_{\rho}^{(n-|\lambda|, \lambda)} \chi_{\rho}^{(n-|\mu|, \mu)} \chi_{\rho}^{(n-|\nu|, \nu)} / z_{\rho}
$$

stabilize as $n \rightarrow \infty$. A lower bound for $n$ involving the three partitions $\lambda, \mu, \nu$ beyond which this stabilization occurs was identified by Vallejo [Va] as $n \geq \max \left\{\mu_{1}+|\mu|+\right.$ $\left.|\lambda|, \nu_{1}+|\nu|+|\lambda|, 2|\lambda|\right\}$. We give next a lower bound involving only the two partitions indexing a product of character polynomials not as sharp but sufficient for our needs.

Proposition 4.2 The stable values of the coefficients $c_{(n-|\lambda|, \lambda),(n-|\mu|, \mu),(n-|\nu|, \nu)}$ are the coefficients $d_{\lambda, \mu, \nu}$ in the expansion

$$
q_{\mu}(x) q_{\nu}(x)=\sum_{|\lambda| \leq|\mu|+|\nu|} d_{\lambda, \mu, \nu} q_{\lambda}(x)
$$

and this value is reached as soon as $n \geq 2(|\mu|+|\nu|)$

Proof. Since the product $q_{\mu}(x) q_{\nu}(x)$ is of partition degree $|\mu|+|\nu|$, from our Remark 4.1 it follows that the character polynomials involved in the expansion of $q_{\mu}(x) q_{\nu}(x)$ are the $q_{\lambda}(x)$ with $|\lambda| \leq|\mu|+|\nu|$ as asserted in equation 4.11. Now, the condition $n \geq 2(|\mu|+|\nu|)$ assures that we have the three inequalities

$$
n-|\lambda| \geq|\lambda| \geq \lambda_{1}, \quad n-|\mu| \geq|\mu| \geq \mu_{1}, \quad n-|\nu| \geq|\nu| \geq \nu_{1} .
$$

From this, and equation I.2 it follows that $q_{\lambda}\left(a_{1}, \ldots, a_{n}\right)=\chi_{\alpha}^{(n-|\lambda|, \lambda)}, q_{\mu}\left(a_{1}, \ldots, a_{n}\right)=$ $\chi_{\alpha}^{(n-|\mu|, \mu)}, q_{\nu}\left(a_{1}, \ldots, a_{n}\right)=\chi_{\alpha}^{(n-|\nu|, \nu)}$. Thus we can use proposition 4.1 and derive that $d_{\lambda, \mu, \nu}=\left\langle\left\langle q_{\mu} q_{\nu}, q_{\lambda}\right\rangle\right\rangle=\sum_{\alpha \vdash n} \frac{\chi_{\alpha}^{(n-|\mu|, \mu)} \chi_{\alpha}^{(n-|\nu|, \nu)} \chi_{\alpha}^{(n-|\lambda|, \lambda)}}{1^{a_{1}} 2^{a_{2}} \ldots n^{a_{n}} a_{1} ! a_{2} ! \ldots a_{n} !}=c_{(n-|\lambda|, \lambda),(n-|\mu|, \mu),(n-|\nu|, \nu)}$

$\forall n \geq 2(|\mu|+|\nu|)$, completing our proof. 
This result has a sharper and more general version that involves signed Schur functions and that can be stated as follows :

Theorem 4.1 For $\mu^{(1)} \vdash k_{1}, \mu^{(2)} \vdash k_{2}, \ldots, \mu^{(r)} \vdash k_{r}$ we have

$$
s_{n-k_{1}, \mu^{(1)}} * s_{n-k_{2}, \mu^{(2)}} * \cdots * s_{n-k_{r}, \mu^{(r)}}=\sum_{|\lambda| \leq k_{1}+k_{2}+\cdots+k_{r}} d_{\lambda ; \mu^{(1)}, \mu^{(2)}, \ldots, \mu^{(r)}} s_{(n-|\lambda|, \lambda)} \quad \forall n \geq 0
$$

where the coefficients $d_{\lambda ; \mu^{(1)}, \mu^{(2)}, \ldots, \mu^{(r)}}$ are given by the expansion

$$
q_{\mu^{(1)}}(x) q_{\mu^{(2)}}(x) \cdots q_{\mu^{(r)}}(x)=\sum_{|\lambda| \leq k_{1}+k_{2}+\cdots+k_{r}} d_{\lambda ; \mu^{(1)}, \mu^{(2)}, \ldots, \mu^{(r)}} q_{\lambda}(x)
$$

and each factor $s_{n-k_{1}, \mu^{(1)}}, \ldots, s_{n-k_{r}, \mu^{(r)}}$ as well as $s_{n-|\lambda|, \lambda}$ are interpreted as signed Schur functions.

Proof. Proceeding exacltly as we did for $r=2$ we show that the expansion of the product $q_{\mu^{(1)}}(x) q_{\mu^{(2)}}(x) \cdots q_{\mu^{(r)}}(x)$ involves only character polynomials $q_{\lambda}(x)$ with $|\lambda| \leq$ $k_{1}+k_{2}+\cdots+k_{r}$ as asserted in 4.13. It then follows that when $k \geq k_{1}+k_{2}+\cdots+k_{r}$ and $\left\|\left(a_{1}, a_{2}, \ldots, a_{k}\right)\right\|=n$

$$
\left.q_{\mu^{(1)}}(x) q_{\mu^{(2)}}(x) \cdots q_{\mu^{(r)}}(x)\right|_{x \rightarrow\left(a_{1}, a_{2}, \ldots, a_{k}\right)}=\chi_{\alpha}^{\left(n-k_{1}, \mu^{(1)}\right)} \chi_{\alpha}^{\left(n-k_{2}, \mu^{(2)}\right)} \cdots \chi_{\alpha}^{\left(n-k_{r}, \mu^{(r)}\right)}
$$

and 4.13 gives

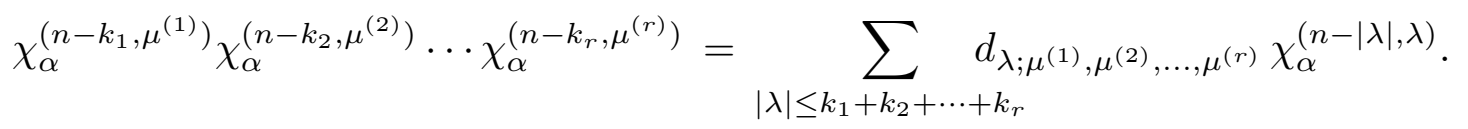

Multiplying by $p_{\alpha} / z_{\alpha}$, summing over all $\alpha \vdash n$ and using equation 3.5 gives equation 4.12.

Remark 4.2. We should note that once the construction of equation 4.13 is implemented on a computer by the two step algorithm given above, then combining with a conversion of equation 4.13 into equation 4.12 can quickly provide the reader with all the tables appearing in the classical Murnaghan paper [Mu].

We should mention that the bound $n \geq 2(|\mu|+|\nu|)$ involving only two partitions is the best we can say without getting into the specifics of the components of the partitions $\mu$ and $\nu$. For example, from $q_{2}=x_{2}-\frac{3}{2} x_{1}+\frac{1}{2} x_{1}^{2}$ and $q_{1}=x_{1}-1$ our two step algorithm gives

$$
q_{2} q_{1}=q_{1}+q_{2}+q_{1,1}+q_{2,1}+q_{3}
$$


with

$q_{1,1}=1-\frac{3}{2} x_{1}+\frac{1}{2} x_{1}^{2}-x_{2}, q_{2,1}=\frac{1}{3} x_{1}^{3}-2 x_{1}^{2}+\frac{8}{3} x_{1}-x_{3}, q_{3}=\frac{1}{6} x_{1}^{3}-x_{1}^{2}+\frac{5}{6} x_{1}-x_{2}+x_{1} x_{2}+x_{3}$.

Now we see from this that although $q_{2} q_{1} \in Q\left[x_{1}, x_{2}\right]$, the expansion equation 4.15 lies in $Q\left[x_{1}, x_{2}, x_{3}\right]$. This fact is responsible for the stabilization of the Kronecker coefficients to take place only after $n \geq 2(2+1)=6$. Indeed, from Theorem 4.1 we derive that equation 4.8 yields the Kronecker product expansion

$$
s_{n-2,2} * s_{n-1,1}=s_{n-1,1}+s_{n-2,2}+s_{n-2,1,1}+s_{n-3,2,1}+s_{n-3,3}
$$

Now this is valid for all values of $n$, provided Schur functions with non partition indexing are straightened out by the commutativity relations in Remark 2.1. To understand why stabilization of Kronecker coefficients may only take place after $n$ is sufficiently large, it is sufficient to see what happens for small values of $n$.

(1) Setting $n=3$ in 4.16 gives

$$
s_{1,2} * s_{2,1}=s_{2,1}+s_{1,2}+s_{1,1,1}+s_{0,2,1}+s_{0,3}=s_{2,1}-s_{1,2}+s_{1,1,1}-s_{1,1,1}-s_{2,1}=0
$$

since $s_{12}=-s_{12}=0$. So we have $0=0$ here.

(2) Setting $n=4$ in equation 4.16 gives

$$
\begin{aligned}
s_{2,2} * s_{3,1} & =s_{3,1}+s_{2,2}+s_{2,1,1}+s_{1,2,1}+s_{1,3}=s_{3,1}+s_{2,2}+s_{2,1,1}-s_{1,2,1}-s_{2,2} \\
& =s_{3,1}+s_{2,1,1}
\end{aligned}
$$

since $s_{1,2,1}=-s_{1,2,1}=0$ and the Kronecker coefficient $\left\langle s_{n-2,2} * s_{n-1,1}, s_{n-2,2}\right\rangle$ is not stabilized yet because the corresponding term has been eliminated by the term $s_{4-3,3}=-s_{2,2}$

(3) Setting $n=5$ in equation 4.16 gives

$$
\begin{aligned}
s_{3,2} * s_{4,1} & =s_{4,1}+s_{3,2}+s_{3,1,1}+s_{2,2,1}+s_{2,3}=s_{4,1}+s_{3,2}+s_{3,1,1}+s_{2,2,1}-s_{2,3} \\
& =s_{4,1}+s_{3,2}+s_{3,1,1}+s_{2,2,1}
\end{aligned}
$$

since $s_{2,3}=-s_{2,3}=0$. This result is again correct even though the term $s_{n-3,3}$ still yields no contribution. On the other hand setting $n=6$ in equation 4.16 gives $s_{4,2} * s_{5,1}=s_{5,1}+s_{4,2}+s_{4,1,1}+s_{3,2,1}+s_{3,3}$ and all the terms survive since all Schur functions are indexed with partitions. 
So we observed that in this example most of the stabilization failed to happen when $n<6$ because the term $s_{(n-3,3)}$ in equation 4.16 , which comes from $q_{3}$ in equation 4.8 , pops out only in the second step of the algorithm. In summary, to assure that equation 4.12 remains true, the straightening of terms $s_{n-|\lambda|, \lambda}$ causes terms with partition indexing to disappear from the right-hand side, and this is precisely what causes the delay in the stabilization of the Kronecker coefficients.

\section{The Hecke algebra case}

In [Ra] Arun Ram obtains a $q$-analog of the Frobenius formula in equation I.2 from which he derives an algorithm for the construction of the irreducible characters of the Hecke Algebra $\mathcal{H}_{n}(q)$. Ram's formula, in our notation, may be written in the form

$$
\chi_{\alpha}^{\lambda}(q)=\left.\frac{1}{(q-1)^{\ell(\alpha)}} h_{\alpha}\left[X_{n}(q-1)\right]\right|_{s_{\lambda}\left[X_{n}\right]}
$$

In spite of its rational presentation, this definition yields a polynomial $\chi_{\alpha}^{\lambda}(q)$ which gives the value of the irreducible character of $\mathcal{H}_{n}(q)$ indexed by $\lambda$, on some special elements of $\mathcal{H}_{n}(q)$ determined from the partition $\alpha$. Ram shows that all the other character values are obtained as linear combinations of the polynomials $\chi_{\alpha}^{\lambda}(q)$. Remarkably, Ram is also able to show that the polynomials $\chi_{\alpha}^{\lambda}(q)$ may be computed manually by an algorithm that is essentially a $q$-analog of the Murnaghan-Nakayama rule.

The methods we developped in the $S_{n}$ case can also be extended to $\mathcal{H}_{n}(q)$ thereby obtaining an explicit, albeit not as simple, $q$-analog of the character polynomial $q_{\mu}\left(x_{1}, x_{2}, \ldots, x_{k}\right)$. Before we can state our result, we need a few preliminary observations.

Proposition 5.1 For each partition $\mu \vdash k$ and integer $n \geq k$ we have

$$
\chi_{\alpha}^{(n-k, \mu)}(q)=\frac{1}{(q-1)^{\ell(\alpha)}}\left\langle s_{(n-k, \mu)}\left[X_{n}(q-1)\right], h_{\alpha}\left[X_{n}\right]\right\rangle .
$$

Proof. From the Cauchy formula for two alphabets $X_{n}$ and $Y_{n}(q-1)$ we derive that

$$
\sum_{\alpha \vdash n} h_{\alpha}\left[X_{n}(q-1)\right] m_{\alpha}\left[Y_{n}\right]=h_{n}\left[X_{n}(q-1) Y_{n}\right]=\sum_{\lambda \vdash n} s_{\lambda}\left[X_{n}\right] s_{\lambda}\left[Y_{n}(q-1)\right] .
$$

Thus equating coefficients of $s_{\lambda}\left[X_{n}\right]$ on both sides of 5.3 and using 5.1 gives

$$
\sum_{\alpha \vdash n}(q-1)^{\ell(\alpha)} \chi_{\alpha}^{\lambda}(q) m_{\alpha}\left[Y_{n}\right]=s_{\lambda}\left[Y_{n}(q-1)\right]
$$


Recalling that $h_{\alpha}\left[Y_{n}\right]$ and $m_{\alpha}\left[Y_{n}\right]$ are dual basis elements with respect to the Hall inner product, we immediately derive that

$$
(q-1)^{\ell(\alpha)} \chi_{\alpha}^{\lambda}(q)=\left\langle s_{\lambda}\left[Y_{n}(q-1)\right], h_{\alpha}\left[Y_{n}\right]\right\rangle .
$$

and equation 5.2 is obtained by setting $\lambda=(n-k, \mu)$.

Formula 5.2 brings us again in a position to use the identity 2.8 and remark 2.1. More precisely, making the substitution $X_{n} \rightarrow X_{n}(q-1)$ in equation 2.8 we obtain that

$$
s_{(n-k, \mu)}\left[X_{n}(q-1)\right]=\left.\Omega\left[z X_{n}(q-1)\right] s_{\mu}\left[X_{n}(q-1)-1 / z\right]\right|_{z^{n-k}},
$$

and this brings equation 5.2 to the form

$$
\left.\chi_{\alpha}^{(n-k, \mu)}(q)=\frac{1}{(q-1)^{\ell(\alpha)}}\left\langle\Omega\left[z X_{n}(q-1)\right] s_{\mu}\left[X_{n}(q-1)-1 / z\right]\right], h_{\alpha}\left[X_{n}\right]\right\rangle\left.\right|_{z^{n-k}} .
$$

To show that this expression is a polynomial in $a_{1}, a_{2}, \ldots, a_{n}$ and to identify it we need some auxiliary facts.

Proposition 5.2 Let $A\left[X_{n}\right]$ be a homogeneous symmetric polynomial of degree $m$ and let

$Q_{A}\left(h_{1}, \ldots, h_{m}\right)$ be the polynomial giving the expansion of $A$ in terms of the homogeneous basis. Then

$$
\left\langle h_{m}\left[X_{n}(q-1)\right], A\left[X_{n}\right]\right\rangle=\left.Q_{A}\left(h_{1}, h_{2}, \ldots, h_{m}\right)\right|_{h_{i} \rightarrow q^{i-1}(q-1)} .
$$

Proof. Clearly it is sufficient to prove equation 5.8 when $A=h_{\beta}$ with $|\beta|=m$. Now in this case we have

$$
\left\langle h_{m}\left[X_{n}(q-1)\right], h_{\beta}\left[X_{n}\right]\right\rangle=\sum_{|\gamma|=m} h_{\gamma}[q-1]\left\langle m_{\gamma}\left[X_{n}\right], h_{\beta}\left[X_{n}\right]\right\rangle=h_{\beta}[q-1],
$$

where the last equality is again due to the fact that the two bases $\left\{m_{\beta}\left[X_{n}\right]\right\}_{\beta}$ and $\left\{h_{\beta}\left[X_{n}\right]\right\}_{\beta}$ are dual with respect to the Hall inner product. However it is easily verified that for any $i \geq 1$ we have

$$
h_{i}[q-1]=q^{i-1}(q-1) .
$$

thus equation 5.9 is none other than

$$
\left\langle h_{m}\left[X_{n}(q-1)\right], h_{\beta}\left[X_{n}\right]\right\rangle=\left.h_{\beta}\right|_{h_{i} \rightarrow q^{i-1}(q-1)} .
$$

THE EleCtronic journal of COMbinatorics 16(2) (2009), \#R19 
This establishes equation 5.8 for $A=h_{\beta}$ and completes our proof.

Proposition 5.3 If $A$ is a homogeneous symmetric polynomial of degree $k$ and $|\alpha|=n$ then

$$
\begin{aligned}
\left.\left\langle\Omega\left[z X_{n}(q-1)\right] A\left[X_{n}(q-1)-1 / z\right], h_{\alpha}\left[X_{n}\right]\right\rangle\right|_{z^{n-k}} \\
=\left.A\left[X_{n}(q-1)-1\right]^{\perp} h_{\alpha}\left[X_{n}\right]\right|_{h_{i} \rightarrow q^{i-1}(q-1)}
\end{aligned}
$$

here it is understood that the substitution $h_{i} \rightarrow q^{i-1}(q-1)$ must be carried out after the polynomial $A\left[X_{n}(q-1)-1\right]^{\perp} h_{\alpha}\left[X_{n}\right]$ has been expanded in the homogeneous basis. Proof. Setting

$$
A\left[X_{n}(q-1)-1 / z\right]=\sum_{s=0}^{k} A_{s}\left[X_{n}(q-1)\right](-1 / z)^{k-s}
$$

in the right hand side of equation 5.11 gives

$$
\begin{aligned}
\left\langle\Omega\left[z X_{n}(q-1)\right]\right. & \left.A\left[X_{n}(q-1)-1 / z\right], h_{\alpha}\left[X_{n}\right]\right\rangle\left.\right|_{z^{n-k}} \\
& =\left.\sum_{s=0}^{k}\left\langle\Omega\left[z X_{n}(q-1)\right] A_{s}\left[X_{n}(q-1)\right](-1 / z)^{k-s}, h_{\alpha}\left[X_{n}\right]\right\rangle\right|_{z^{n-k}} \\
(\text { by 1.3) } & =\sum_{s=0}^{k}(-1)^{k-s}\left\langle h_{n-s}\left[X_{n}(q-1)\right] A_{s}\left[X_{n}(q-1)\right], h_{\alpha}\left[X_{n}\right]\right\rangle \\
& =\sum_{s=0}^{k}(-1)^{k-s}\left\langle h_{n-s}\left[X_{n}(q-1)\right], A_{s}\left[X_{n}(q-1)\right]^{\perp} h_{\alpha}\left[X_{n}\right]\right\rangle
\end{aligned}
$$

(by proposition 5.2) $=\left.\sum_{s=0}^{k}(-1)^{k-s} A_{s}\left[X_{n}(q-1)\right]^{\perp} h_{\alpha}\left[X_{n}\right]\right|_{h_{i} \rightarrow q^{i-1}(q-1)}$

$$
=\left.\left(\sum_{s=0}^{k}(-1)^{k-s} A_{s}\left[X_{n}(q-1)\right]\right)^{\perp} h_{\alpha}\left[X_{n}\right]\right|_{h_{i} \rightarrow q^{i-1}(q-1)}
$$

$$
\text { (by 5.12) }=\left.A\left[X_{n}(q-1)-1\right]^{\perp} h_{\alpha}\left[X_{n}\right]\right|_{h_{i} \rightarrow q^{i-1}(q-1)} \quad \text { Q.E.D. }
$$

The last ingredient needed to establish that $\chi_{\alpha}^{(n-k, \mu)}(q)$ is a polynomial in $a_{1}, a_{2}, \ldots, a_{n}$ is given by the following interesting identity. 
Proposition 5.4 For every pair of integers $m, i \geq 1$ we have

$$
i \partial_{p_{i}} h_{m}= \begin{cases}h_{m-i} & \text { if } m \geq i \\ 1 & \text { if } m=i \\ 0 & \text { if } m<i\end{cases}
$$

Proof. It is well known and easy to show that

$$
\text { a) } i \partial_{p_{i}}=p_{i}^{\perp}, \quad \text { b) } h_{i}^{\perp} h_{m}= \begin{cases}h_{m-i} & \text { if } m \geq i \\ 1 & \text { if } m=i \\ 0 & \text { if } m<i .\end{cases}
$$

Since $p_{1}=h_{1}$ we see that equation 5.13 holds true for $i=1$. We can thus proceed by induction on $i$. So suppose that 5.13 holds true for $1 \leq i \leq k-1$. Taking the logarithmic derivative of the identity $\sum_{m \geq 0} z^{m} h_{m}=\exp \left(\sum_{k \geq 1} \frac{p_{k}}{k} z^{k}\right)$ established in equation 1.3 and equating coefficients of $z^{k}$ gives the Newton identity $k h_{k}=p_{k}+p_{k-1} h_{1}+p_{k-2} h_{2}+$ $\cdots+p_{1} h_{k-1}$. This implies that

$$
k h_{k}^{\perp} h_{m}=p_{k}^{\perp} h_{m}+p_{k-1}^{\perp} h_{1}^{\perp} h_{m}+p_{k-2}^{\perp} h_{2}^{\perp} h_{m}+\cdots+p_{1}^{\perp} h_{k-1}^{\perp} h_{m} .
$$

Thus, using the convention $h_{a}=0$ when $a<0$, the inductive hypothesis and equation 5.14 give for all $m$ :

$$
k h_{m-k}=p_{k}^{\perp} h_{m}+(k-1) h_{m-k}
$$

that is $p_{k}^{\perp} h_{m}=h_{m-k}$ and 5.14 a) for $i=k$ completes the induction and our proof.

Our final step in this section is to use these propositions to evaluate the right hand side of equation 5.7. However, it is quite interesting to see what we obtain in the simplest case $\mu=(1)$.

\section{Theorem 5.1}

$$
\chi_{\alpha}^{(n-1,1)}(q)=a_{1} q^{n-\ell(\alpha)-1}+\ell(\alpha)(q-1) q^{n-\ell(\alpha)-1}-q^{n-\ell(\alpha)}
$$

Proof. Setting $\mu=(1)$ in equation 5.7 and using equation 5.11, we have

$$
\begin{aligned}
\chi_{\alpha}^{(n-1,1)}(q) & =\left.\frac{1}{(q-1)^{\ell(\alpha)}}\left\langle\Omega\left[z X_{n}(q-1)\right]\left(p_{1}(q-1)-1 / z\right), h_{\alpha}\left[X_{n}\right]\right\rangle\right|_{z^{n-k}} \\
& =\left.\frac{1}{(q-1)^{\ell(\alpha)}}\left((q-1) \partial_{p_{1}}-1\right) h_{\alpha}\right|_{h_{i} \rightarrow q^{i-1}(q-1)}
\end{aligned}
$$


Now note that for $\alpha=1^{a_{1}} 2^{a_{2}} \cdots n^{a_{n}}$ we get

$$
\begin{aligned}
\partial_{p_{1}} h_{\alpha} & =\sum_{i=1}^{n} a_{i} \frac{h_{\alpha}}{h_{i}} \partial_{p_{1}} h_{i} \\
(\text { by } 5.14) & =h_{\alpha}\left(a_{1} \frac{1}{h_{1}}+\sum_{i=2}^{n} a_{i} \frac{1}{h_{i}} h_{i-1}\right),
\end{aligned}
$$

and since

$$
\left.h_{\alpha}\right|_{h_{i} \rightarrow q^{i-1}(q-1)}=\prod_{i=1}^{n}\left(q^{i-1}(q-1)\right)^{a_{i}}=q^{n-\ell(\alpha)}(q-1)^{\ell(\alpha)},
$$

equation 5.16 becomes

$$
\begin{aligned}
\chi_{\alpha}^{(n-1,1)}(q) & =\left.\frac{h_{\alpha}}{(q-1)^{\ell(\alpha)}}\left(a_{1} \frac{q-1}{h_{1}}+\sum_{i=2}^{n} a_{i} \frac{q-1}{h_{i}} h_{i-1}-1\right)\right|_{h_{i} \rightarrow q^{i-1}(q-1)} \\
& =q^{n-\ell(\alpha)}\left(a_{1} \frac{q-1}{q-1}+\sum_{i=2}^{n} a_{i} \frac{q-1}{q}-1\right) \\
& =a_{1} q^{n-\ell(\alpha)}+(q-1) q^{n-\ell(\alpha)-1}\left(\ell(\alpha)-a_{1}\right)-q^{n-\ell(\alpha)}
\end{aligned}
$$

This proves equation 5.15.

The polynomiality of $\chi_{\alpha}^{(n-k, \mu)}(q)$ is a special case of the following general fact. Proposition 5.5 For each homgeneous symmetric polynomial $A\left[X_{n}\right]$ of degree $k \leq n$, the function

$$
Q_{A}(a ; q)=\left.\frac{1}{(q-1)^{\ell(\alpha)}} A\left[X_{n}(q-1)-1\right]^{\perp} \prod_{i=1}^{n} h_{i}^{a_{i}}\left[X_{n}\right]\right|_{h_{i} \rightarrow q^{i-1}(q-1)}
$$

is a polynomial in $q$ and $a_{1}, a_{2}, \ldots, a_{n}$ with coefficients depending on $\ell(\alpha)$.

Proof. It is sufficient to prove this for the power basis elements $p_{\theta}=\prod_{i=1}^{k} p_{i}^{t_{i}}$ with $|\theta|=k$. We have

$$
Q_{p_{\theta}}(a ; q)=\left.\frac{1}{(q-1)^{\ell(\alpha)}}\left(\prod_{i=1}^{k}\left(p_{i}\left(q^{i}-1\right)-1\right)^{t_{i}}\right)^{\perp} \prod_{i=1}^{n} h_{i}^{a_{i}}\right|_{h_{i} \rightarrow q^{i-1}(q-1)}
$$

from equation 5.14 a) it follows that

$$
Q_{p_{\theta}}(a ; q)=\left.\frac{1}{(q-1)^{\ell(\alpha)}}\left(\prod_{i=1}^{k}\left(i \partial_{p_{i}}\left(q^{i}-1\right)-1\right)^{t_{i}}\right) \prod_{i=1}^{n} h_{i}^{a_{i}}\right|_{h_{i} \rightarrow q^{i-1}(q-1)} .
$$


Now the first product has an expansion of the form

$\frac{1}{(q-1)^{\ell(\alpha)}} \prod_{i=1}^{k}\left(i \partial_{p_{i}}\left(q^{i}-1\right)-1\right)^{t_{i}}=\sum_{|\beta| \leq k} c_{\beta}(\theta)\left(\frac{-1}{q-1}\right)^{\ell(\alpha)-\ell(\beta)} \prod_{j=1}^{\ell(\beta)}\left(\frac{q^{j}-1}{q-1}\right)^{b_{j}} j^{b_{j}} \partial_{p_{i}}^{b_{j}}$.

where $c_{\beta}(\theta)$ are the coefficients of this expansion. Using this and setting $[j]_{q}=\frac{1-q^{j}}{1-q}=$ $1+q+\cdots q^{j-1}$ the identity in 5.19 becomes

$$
Q_{p_{\theta}}(a ; q)=\left.\sum_{|\beta| \leq k} c_{\beta}(\theta)\left(\frac{-1}{q-1}\right)^{\ell(\alpha)-\ell(\beta)}\left(\prod_{j=1}^{\ell(\beta)}\left(j[j]_{q}\right)^{b_{j}}\right) \prod_{j=1}^{\ell(\beta)} \partial_{p_{j}}^{b_{j}} \cdot \prod_{i=1}^{n} h_{i}^{a_{i}}\right|_{h_{i} \rightarrow q^{i-1}(q-1)} .
$$

This given we are left to show that the desired polynomiality properties are satisfied by each of the expressions

$$
\left.\left(\frac{-1}{q-1}\right)^{\ell(\alpha)-\ell(\beta)} \prod_{j=1}^{\ell(\beta)} \partial_{p_{j}}^{b_{j}} \cdot \prod_{i=1}^{n} h_{i}^{a_{i}}\right|_{h_{i} \rightarrow q^{i-1}(q-1)}
$$

To this end note first that carrying out the differentiations in equation 5.21 and using equation $5.14 \mathrm{~b}$ ) we obtain an expansion of the form

$$
\prod_{j=1}^{\ell(\beta)} \partial_{p_{j}}^{b_{j}} \cdot \prod_{i=1}^{n} h_{i}^{a_{i}}=\sum_{\ell(\delta)=\ell(\alpha)-\ell(\beta)} c_{\delta, \beta}(\alpha) \prod_{i=1}^{\ell(\alpha)-\ell(\beta)} h_{i}^{d_{i}}
$$

with coefficients $c_{\delta, \beta}(\alpha)$ polynomials in $a_{1}, a_{2}, \ldots, a_{n}$. Using this in equation 5.21 we get

$$
\begin{aligned}
\left(\frac{-1}{q-1}\right)^{\ell(\alpha)-\ell(\beta)} & \left.\left.\prod_{j=1}^{\ell(\beta)} \partial_{p_{j}}^{b_{j}} \cdot \prod_{i=1}^{n} h_{i}^{a_{i}}\right|_{h_{i} \rightarrow q^{i-1}(q-1)} c_{\delta, \beta}(\alpha) \prod_{i=1}^{\ell(\alpha)-\ell(\beta)}\left(\frac{-h_{i}}{q-1}\right)^{d_{i}}\right|_{h_{i} \rightarrow q^{i-1}(q-1)} \\
& =\sum_{\ell(\delta)=\ell(\alpha)-\ell(\beta)} c_{\delta, \beta}(\alpha) \prod_{i=1}^{\ell(\delta)}\left(-q^{i-1}\right)^{d_{i}} \\
& =\sum_{\ell(\delta)=\ell(\alpha)-\ell(\beta)} c_{\delta, \beta}(\alpha)(-1)^{\ell(\delta)} q^{|\delta|-\ell(\delta)} \\
& =\sum_{\ell(\delta)=\ell(\alpha)-\ell(\beta)}
\end{aligned}
$$


and equation 5.20 becomes

$$
Q_{p_{\theta}}(a ; q)=\sum_{|\beta| \leq k} c_{\beta}(\theta)\left(\prod_{j=1}^{\ell(\beta)}\left(j[j]_{q}\right)^{b_{j}}\right) \sum_{\ell(\delta)=\ell(\alpha)-\ell(\beta)} c_{\delta, \beta}(\alpha)(-1)^{\ell(\delta)} q^{|\delta|-\ell(\delta)}
$$

and this completes our argument.

\begin{tabular}{|c|c|}
\hline $\begin{array}{c}\chi_{\alpha}^{(n-1,1)}= \\
\chi_{\alpha}^{(n-1,1)}(q)=\end{array}$ & $\begin{array}{l}a_{1}-1 \\
q^{n-\ell-1}\left(a_{1}+(\ell-1)(q-1)-1\right)\end{array}$ \\
\hline $\begin{aligned} \chi_{\alpha}^{(n-2,2)} & = \\
\chi_{\alpha}^{(n-2,2)}(q) & =\end{aligned}$ & $\begin{array}{l}\frac{1}{2}\left(2 a_{2}+a_{1}\left(a_{1}-3\right)\right) \\
\frac{q^{n-\ell-2}}{2}\left(2 q a_{2}+q^{2} a_{1}\left(a_{1}-3\right)+\left(\ell-a_{1}\right)(q-1)\left((q+1) a_{1}+(q-1)(\ell-1)\right)\right)\end{array}$ \\
\hline $\begin{aligned} \chi_{\alpha}^{(n-2,1,1)} & = \\
\chi_{\alpha}^{(n-2,1,1)}(q) & =\end{aligned}$ & $\begin{array}{l}\frac{1}{2}\left(-2 a_{2}+a_{1}^{2}-3 a_{1}+2\right) \\
\frac{q^{n-\ell-2}}{2}\left(-2 a_{2}+q^{2}\left(a_{1}-1\right)\left(a_{1}-2\right)+\left(\ell-a_{1}\right)(q-1)\left((q+1)\left(a_{1}-2\right)+(q-1)(\ell-1)\right)\right)\end{array}$ \\
\hline $\begin{aligned} \chi_{\alpha}^{(n-3,3)} & = \\
\chi_{\alpha}^{(n-3,3)}(q) & =\end{aligned}$ & $\begin{array}{l}\frac{1}{6}\left(6 a_{3}+6 a_{2}\left(a_{1}-1\right)+a_{1}^{3}-6 a_{1}^{2}+5 a_{1}\right)=\frac{1}{6}\left(6 a_{3}+6 a_{2}\left(a_{1}-1\right)+a_{1}\left(a_{1}-1\right)\left(a_{1}-5\right)\right) \\
\frac{q^{n-\ell-3}}{6}\left(6 q^{2} a_{3}+6 q a_{2}\left(a_{1}+(q-1)(\ell-1)-q^{2}\right)+a_{1}^{3}+3 a_{1}^{2}\left((q-1)(\ell-1)-2 q^{2}\right)\right. \\
\left.+a_{1}\left(3 \ell(q-1)\left((q-1)(l-1)-2 q^{2}+1\right)+6 q^{3}-3 q+2\right)+\ell(\ell-1)(q-1)((\ell-1)(q-1)+2 q+1)\right)\end{array}$ \\
\hline $\begin{aligned} \chi_{\alpha}^{(n-3,2,1)} & = \\
\chi_{\alpha}^{(n-3,2,1)}(q) & =\end{aligned}$ & $\begin{array}{l}\frac{1}{3}\left(-3 a_{3}+a_{1}^{3}-6 a_{1}^{2}+8 a_{1}\right)=\frac{1}{3}\left(-3 a_{3}+a_{1}\left(a_{1}-2\right)\left(a_{1}-4\right)\right) \\
\frac{q^{n-\ell-3}}{3}\left(-3 q a_{3}+3(q-1) a_{2}\left[(q-1)(\ell-1)+a_{1}\right]+a_{1}^{3}+3 a_{1}^{2}\left[(q-1)(\ell-1)-\left(q^{2}+1\right)\right]\right. \\
\left.+a_{1}\left[3 \ell(q-1)(\ell(q-1)-q(q+2))+3 q^{2}(2 q+1)-1\right]+\ell(\ell-1)(q-1)^{2}[(\ell-1)(q-1)-q-2]\right)\end{array}$ \\
\hline $\begin{aligned} \chi_{\alpha}^{\left(n-3,1^{3}\right)} & = \\
\chi_{\alpha}^{\left(n-3,1^{3}\right)}(q) & =\end{aligned}$ & $\begin{array}{l}\frac{1}{6}\left(6 a_{3}-6 a_{2}\left(a_{1}-1\right)+a_{1}^{3}-6 a_{1}^{2}+11 a_{1}-6\right)=\frac{1}{6}\left(6 a_{3}-6 a_{2}\left(a_{1}-1\right)+\left(a_{1}-1\right)_{3}\right) \\
\frac{q^{n-\ell-3}}{6}\left(6 a_{3}-6 a_{2}\left[(q-1)(\ell-1)+a_{1}-1\right]+a_{1}^{3}+3 a_{1}^{2}[(q-1)(\ell-1)-2]\right. \\
+a_{1}[3(\ell-1)(q-1)((\ell-2)(q-1)-5)+11]+(\ell-1)_{3}(q-1)^{3} \\
-9(\ell-1)(q-1)[(\ell-1)(q-1)-q-1]-6)\end{array}$ \\
\hline
\end{tabular}

Table 5.1 Character polynomials of $S_{n}$ and $\mathcal{H}_{n}(q)$

Theorem 5.2 For $\mu \vdash k$, the $q$-character value $\chi_{\alpha}^{(n-k, \mu)}(q)$ is a polynomial in $q$ and $a_{1}, a_{2}, \ldots, a_{n}$ with coefficients depending on $n$ and $\ell(\alpha)$. To be precise, if $Q_{p_{\theta}}(a ; q)$ is given by equation 5.23, $\chi_{\alpha}^{(n-k, \mu)}(q)$ may be computed from the identity

$$
\chi_{\alpha}^{(n-k, \mu)}(q)=\sum_{|\theta|=k} \frac{\chi_{\theta}^{\mu}}{z_{\theta}} Q_{p_{\theta}}(a ; q)
$$


Proof. From equation 5.7 and Proposition 5.3 we get

$$
\begin{aligned}
\chi_{\alpha}^{(n-k, \mu)}(q) & \left.=\frac{1}{(q-1)^{\ell(\alpha)}}\left\langle\Omega\left[z X_{n}(q-1)\right] s_{\mu}\left[X_{n}(q-1)-1 / z\right]\right], h_{\alpha}\left[X_{n}\right]\right\rangle\left.\right|_{z^{n-k}} \\
& =\left.\frac{1}{(q-1)^{\ell(\alpha)}} s_{\mu}\left[X_{n}(q-1)-1\right]^{\perp} h_{\alpha}\left[X_{n}\right]\right|_{h_{i} \rightarrow q^{i-1}(q-1)} .
\end{aligned}
$$

and the Frobenius formula in equation 1.21 gives

$$
\begin{aligned}
\chi_{\alpha}^{(n-k, \mu)}(q) & =\left.\frac{1}{(q-1)^{\ell(\alpha)}} \sum_{|\theta|=k} \frac{\chi_{\theta}^{\mu}}{z_{\theta}} p_{\theta}\left[X_{n}(q-1)-1\right]^{\perp} h_{\alpha}\left[X_{n}\right]\right|_{h_{i} \rightarrow q^{i-1}(q-1)} \\
& =\left.\sum_{|\theta|=k} \frac{\chi_{\theta}^{\mu}}{z_{\theta}} \frac{1}{(q-1)^{\ell(\alpha)}}\left(\prod_{i=1}^{k}\left(p_{i}\left(q^{i}-1\right)-1\right)^{t_{i}}\right)^{\perp} h_{\alpha}\left[X_{n}\right]\right|_{h_{i} \rightarrow q^{i-1}(q-1)}
\end{aligned}
$$

and equation 5.24 follows from equation 5.18. Table 5.1, where $\ell$ stands for $\ell(\alpha)$, should dispell any doubts that the polynomials yielding the q-character values $\chi_{\alpha}^{(n-k, \mu)}(q)$ are natural q-analogs of the character polynomials.

\section{Set polynomials and applications}

We begin by noting that, using equation 2.8 or I.2, the identity 2.3 may be rewritten in any of the following two equivalent forms:

$$
\begin{gathered}
\left\langle\mathbf{H}_{n-k} s_{\mu}, p_{\alpha}\right\rangle=\left\langle\mathcal{T}_{-1} s_{\mu}, \mathcal{T}_{1} p_{\alpha}\right\rangle . \\
\mathbf{q}_{s_{\mu}}\left(a_{1}, a_{2}, \ldots, a_{n}\right)=\left\langle\mathcal{T}_{-1} s_{\mu}, \mathcal{T}_{1} p_{\alpha}\right\rangle .
\end{gathered}
$$

Since both $\left\{p_{\alpha}\right\}_{\alpha}$ and $\left\{s_{\mu}\right\}_{\mu}$ are linear bases of $\Lambda$, equation 6.1 extends to all pairs of homogeneous symmetric functions $f, g$ of respective degrees $k$ and $n$ :

$$
\left\langle\mathbf{H}_{n-k} f, g\right\rangle=\left\langle\mathcal{T}_{-1} f, \mathcal{T}_{1} g\right\rangle .
$$

It develops that the operator $\mathbf{H}_{n-k}$ has a companion operator $\mathbf{h}_{\mathbf{n}-\mathbf{k}}$ which is "multiplication by $h_{n-k}$ " with companion identity

$$
\left\langle\mathbf{h}_{\mathbf{n}-\mathbf{k}} f, g\right\rangle=\left\langle f, \mathcal{T}_{1} g\right\rangle .
$$

To prove this given equation 1.11, we need only check that $\left\langle\mathbf{h}_{\mathbf{n}-\mathbf{k}} f, g\right\rangle=\sum_{m \geq 0}\left\langle h_{m} f, g\right\rangle$. Equation 6.4 becomes a consequence of the identity

$$
\left\langle p_{\alpha}, h_{m}\right\rangle= \begin{cases}1 & \text { if } \alpha \vdash m \\ 0 & \text { otherwise. }\end{cases}
$$


We shall see here the role played by these identities in the computation of some Kronecker products. With the present notation, replacing $s_{\mu}$ by $f$ in 6.2 gives

$$
\mathbf{q}_{f}\left(a_{1}, a_{2}, \ldots, a_{n}\right)=\left\langle\mathcal{T}_{-1} f, \mathcal{T}_{1} p_{\alpha}\right\rangle
$$

To get the companion identity we are led to introduce another map $\mathbf{v}: \Lambda \rightarrow Q\left[x_{1}, x_{2}, \ldots\right]$ defined as the linear extension of the map

$$
\mathbf{v}\left(p_{\beta}\right)=\mathbf{v}_{p_{\beta}}\left(x_{1}, x_{2}, \ldots, x_{k}\right)=\downarrow \prod_{i=1}^{n}\left(i x_{i}\right)^{b_{i}}=\left(\prod_{i=1}^{n} i^{b_{i}}\right) \prod_{i=1}^{n}\left(x_{i}\right)_{b_{i}}=z_{\beta} \prod_{i=1}^{n}\left(\begin{array}{c}
x_{i} \\
b_{i}
\end{array}\right)
$$

This given, 6.4 for $g=p_{\alpha}$ may be rewritten as

$$
\mathbf{v}_{f}\left(a_{1}, a_{2}, \ldots, a_{n}\right)=\left\langle f, \mathcal{T}_{1} p_{\alpha}\right\rangle
$$

To see this we simply note that for $f=p_{\beta} / z_{\beta}$, with $\beta \vdash k, 6.4$ gives

$$
\begin{aligned}
\left\langle p_{\beta} / z_{\beta}, \mathcal{T}_{1} p_{\alpha}\right\rangle & =\left\langle h_{n-k} p_{\beta} / z_{\beta}, p_{\alpha}\right\rangle \\
& =\frac{1}{z_{\beta}}\left\langle h_{n-k}, \prod_{i=1}^{k}\left(i \partial_{p_{i}}\right)^{b_{i}} p_{\alpha}\right\rangle \\
& =\prod_{i=1}^{k}\left(\begin{array}{c}
a_{i} \\
b_{i}
\end{array}\right)\left\langle h_{n-k}, \prod_{i=1}^{n} p_{i}^{a_{i}-b_{i}}\right\rangle \\
& =\prod_{i=1}^{k}\left(\begin{array}{c}
a_{i} \\
b_{i}
\end{array}\right)=\mathbf{v}\left(\frac{p_{\beta}}{z_{\beta}}\right)\left(a_{1}, a_{2}, \ldots, a_{n}\right)
\end{aligned}
$$

this proves 6.8 for all $f \in \Lambda$. For reasons that will soon be apparent we are inclined to call "set polynomials" the polynomials $\mathbf{v}(f)$ defined by equation 6.7 .

It is well known that the number of occurrences of the identity representation in the action of a group $G$ on a family $\mathcal{F}$ of objects is equal to the number of orbits of this action. If $G$ acts transitively then its action on $\mathcal{F}$ is equivalent to its action on the left cosets of the stabilizer of any one of the elements of $\mathcal{F}$. It follows then that if $G=\mathcal{S}_{n}$, the group of permutations of $\Omega_{n}=\{1,2, \ldots, n\}$ and if $\mathcal{F}$ is the family of $r$-subsets of $\Omega_{n}$ then the Frobenius characteristic of the action of $\mathcal{S}_{n}$ on $\mathcal{F}$ is the symmetric function $h_{r} h_{n-r}$. Denoting by $\Pi_{\alpha}^{n-r, r}$ the character of this action evaluated on the permutations of cycle type $\alpha$ we must have

$$
\begin{aligned}
h_{n-r} h_{r} & =\sum_{\alpha \vdash n} \Pi_{\alpha}^{n-r, r} \frac{p_{\alpha}}{z_{\alpha}} \\
& =\sum_{\alpha \vdash n}\left\langle h_{n-r} h_{r}, p_{\alpha}\right\rangle \frac{p_{\alpha}}{z_{\alpha}}
\end{aligned}
$$


Combining equations $6.4,6.8,6.10$ and 6.11 we derive that

$$
\Pi_{\alpha}^{n-r, r}=\left\langle h_{n-r} h_{r}, p_{\alpha}\right\rangle=\mathbf{v}_{h_{r}}\left(a_{1}, a_{2}, \ldots, a_{n}\right)=\sum_{\beta \vdash k} \mathbf{v}_{\frac{p_{\beta}}{z_{\beta}}}\left(a_{1}, a_{2}, \ldots, a_{n}\right)
$$

and 6.9 gives

$$
\Pi_{\alpha}^{n-r, r}=\sum_{\beta \vdash r} \prod_{i=1}^{r}\left(\begin{array}{c}
a_{i} \\
b_{i}
\end{array}\right)
$$

an expression that was called $\pi_{n}(a)$ by Specht in [Sp] and was used to compute character polynomials. It follows then that the Frobenius characteristic of the action of $S_{n}$ on the $k$-fold cartesian product $\mathcal{F} \times \cdots \times \mathcal{F}:=\mathcal{F}^{\times k}$ is given by the $k$-fold Kronecker product

$$
h_{r} h_{n-r} * h_{r} h_{n-r} * \cdots * h_{r} h_{n-r}=\left(h_{r} h_{n-r}\right)^{* k}=\sum_{\alpha \vdash n}\left(\Pi_{\alpha}^{n-r, r}\right)^{k} \frac{p_{\alpha}}{z_{\alpha}}
$$

In particular we see that the scalar product

$$
\left\langle\left(h_{r} h_{n-r}\right)^{* k}, s_{n}\right\rangle=\sum_{\alpha \vdash n}\left(\Pi_{\alpha}^{n-r, r}\right)^{k} \frac{1}{z_{\alpha}}
$$

gives the multiplicity of the identity representation in the action of $\mathcal{S}_{n}$ on the $k$-tuples $\left(A_{1}, \ldots, A_{k}\right)$ of $r$-subsets of $\Omega_{n}$. Thus the Kronecker coefficient in 6.13 yields a count of the number of orbits under this action. Now it is easy to see that an orbit under this action is completely characterized by the vector $\left(n_{1}, n_{2}, \ldots, n_{2^{k}}\right)$ giving the respective cardinalities of the $2^{k}$ regions that decompose $\Omega_{n}$ in the Venn diagram of the $k$-tuple $\left(A_{1}, A_{2}, \ldots, A_{k}\right)$. To be precise, for $1 \leq i \leq 2^{k}$, if $\epsilon_{1} \epsilon_{2} \cdots \epsilon_{k}$ is the binary expansion of $i-1$ with $k$ digits, we set

$$
n_{i}=\operatorname{card}\left(A_{1}^{\epsilon_{1}} \cap A_{2}^{\epsilon_{2}} \cap \cdots \cap A_{k}^{\epsilon_{k}}\right)
$$

where for a subset $A \subseteq \Omega_{n}$ we let

$$
A^{\epsilon}= \begin{cases}A & \text { if } \epsilon=0 \\ \Omega_{n} / A & \text { if } \epsilon=1\end{cases}
$$

Thus the Kronecker coefficient in 6.13 enumerates the number of solutions of the Diophantine system $\mathcal{D}_{k}(n)$ that must be satisfied by the vector $\left(n_{1}, n_{2}, \ldots, n_{2^{k}}\right)$ in order that each of the $A_{i}$ has cardinality $r$ and $\Omega_{n}$ has cardinality $n$. For instance when $k=3$, using the notation in 6.14 , we get 


$$
\mathcal{D}_{3}(n)=\| \begin{aligned}
\operatorname{card}\left(A_{1}\right) & =n_{1}+n_{2}+n_{3}+n_{4}=r \\
\operatorname{card}\left(A_{2}\right) & =n_{1}+n_{2}+n_{5}+n_{6}=r \\
\operatorname{card}\left(A_{3}\right) & =n_{1}+n_{3}+n_{5}+n_{7}=r \\
\operatorname{card}\left(\Omega_{n} / A_{1}\right) & =n_{5}+n_{6}+n_{7}+n_{8}=n-r
\end{aligned}
$$

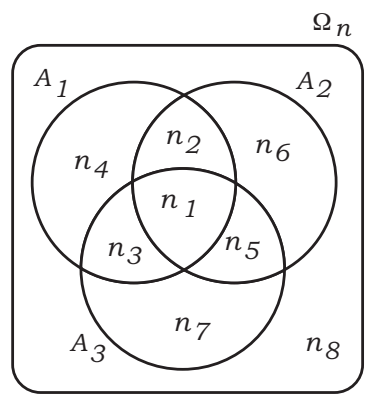

Let us set

$$
F_{k}(x ; r)=\sum_{n \geq r}\left\langle\left(h_{r} h_{n-r}\right)^{* k}, h_{n}\right\rangle x^{n}
$$

where the left hand side of the scalar product is the $k^{t h}$ Kronecker power of $h_{r} h_{n-r}$. As we mentioned above the Kronecker coefficients in 6.16 must stabilize as $n \rightarrow \infty$. This means that we have

$$
F_{k}(x ; r)=\frac{P_{k}(x ; r)}{1-x}
$$

for a suitable polynomial $P_{k}(x ; r) \in \mathbb{N}[x]$.

Using a different but entirely equivalent combinatorial model G. Labelle in [La] showed that the exponential generating function of these polynomials, for $r=2$, has the following beautiful expansion

$$
\sum_{k \geq 0} P_{k}(x ; 2) \frac{y^{k}}{k !}=e^{\frac{x^{2}}{2}\left(e^{y}-1\right)} e^{-x} \sum_{n \geq 0} \frac{x^{n}}{n !} e^{\left(\begin{array}{c}
n \\
2
\end{array}\right) y}
$$

Labelle [La] obtained this result by showing that the polynomial $P_{k}(x ; 2)$ enumerates certain multigraphs with $k$ edges and no loop. Labelle obtains 6.18 by an elegant application of the theory of Species, which motivates a number of manipulations out of which emerges 6.18 in its surprisingly simple final form.

The stabilization reflected by the identity in equation 6.17 can be easily explained combinatorially. More generally if we denote by $\epsilon_{s}(i)$ the $s^{\text {th }}$ digit of the binary expansion of $i-1$ then the diophantine system whose solutions are counted by the Kronecker coefficient in equation 6.16 takes the form

$$
\mathcal{D}_{k}(n)=\| \begin{aligned}
\operatorname{card}\left(A_{1}\right) & =\sum_{i=1}^{2^{k}}\left(1-\epsilon_{1}(i)\right) n_{i}=r \\
\operatorname{card}\left(A_{2}\right) & =\sum_{i=1}^{2^{k}}\left(1-\epsilon_{2}(i)\right) n_{i}=r \\
\cdots & \cdots \quad \cdots \quad \cdots \quad \cdots \\
\operatorname{card}\left(A_{k}\right) & =\sum_{i=1}^{2^{k}}\left(1-\epsilon_{k}(i)\right) n_{i}=r \\
\operatorname{card}\left(\Omega_{n} / A_{1}\right) & =n_{2^{k-1}+1}+n_{2^{k-1}+2}+\cdots+n_{2^{k}}=n-r
\end{aligned}
$$


We can now easily see what happens as $n \rightarrow \infty$. Since $n_{2^{k}}$ occurs only in the last equation, every solution $\left(n_{1}, n_{2}, \ldots, n_{2^{k}}\right)$ of $\mathcal{D}_{k}(n)$ comes from the solution $\left(n_{1}, n_{2}, \ldots, n_{2^{k}-1}, 0\right)$ of $\mathcal{D}_{k}(m)$ for

$$
m=n-n_{2^{k}}=n_{1}+n_{2}+\cdots+n_{2^{k}-1}=\operatorname{card}\left(A_{1} \cup A_{2} \cup \cdots \cup A_{k}\right) \leq k r .
$$

This shows that for any $n$ the solutions to $\mathcal{D}_{k}(n)$ can be simply obtained by adding the component $n_{2^{k}}=n-m$ to a solution $\left(n_{1}, n_{2}, \ldots, n_{2^{k}-1}, 0\right)$ for an $m \leq n$. From this we derive that the number of solutions of the system $\mathcal{D}_{k}(n)$ is simply given by the sum $c_{k}(r)+c_{k}(r+1)+\cdots+c_{k}(\min \{n, k r\})$ where $c_{k}(m)$, for any $r \leq m \leq k r$, denotes the number of solutions $\left(n_{1}, n_{2}, \ldots, n_{2^{k}-1}, 0\right)$ of the system $\mathcal{D}_{k}(m)$. It follows then that we must also have

$$
\left\langle\left(h_{r} h_{n-r}\right)^{* k}, h_{n}\right\rangle=c_{k}(r)+c_{k}(r+1)+\cdots+c_{k}(\min \{n, k r\}) .
$$

This gives that the generating function $F_{k}(x ; r)$ has the form

$$
F_{k}(x ; r)=\frac{\sum_{m=r}^{k r} c_{k}(m) x^{m}}{1-x}
$$

which shows that

$$
P_{k}(x ; r)=\sum_{m=r}^{k r} c_{k}(m) x^{m}
$$

As an application of symmetric function methods in the computation of Kronecker coefficients we now derive a Labelle type formula for the exponential generating function of these polynomials. More precisely we establish the following general result.

Theorem 6.1 Setting for all $r \geq 2$

$$
F_{r}(x, y)=\sum_{k \geq 0} F_{k}(x ; r) \frac{y^{k}}{k !}
$$

we have

$$
F_{r}(x, y)=\frac{e^{-\sum_{s=1}^{r} \frac{x^{s}}{s}}}{1-x} \sum_{a_{1} \geq 0} \sum_{a_{2} \geq 0} \cdots \sum_{a_{r} \geq 0} \frac{\left(\frac{x}{1}\right)^{a_{1}}}{a_{1} !} \frac{\left(\frac{x^{2}}{2}\right)^{a_{2}}}{a_{2} !} \cdots \frac{\left(\frac{x^{r}}{r}\right)^{a_{1}}}{a_{r} !} \exp \left(y \sum_{\beta \vdash r} \prod_{i=1}^{r}\left(\begin{array}{c}
a_{i} \\
b_{i}
\end{array}\right)\right)
$$


Proof. Using equation 6.13 we get that

$$
F_{k}(x ; r)=\sum_{n \geq 0} x^{n} \sum_{\alpha \vdash n}\left(\Pi_{\alpha}^{n-r, r}\right)^{k} \frac{1}{z_{\alpha}} .
$$

Thus

$$
\begin{aligned}
& F_{r}(x, y)=\sum_{n \geq 0} x^{n} \sum_{\alpha \vdash n} e^{y\left(\Pi_{\alpha}^{n-r, r}\right)} \frac{1}{z_{\alpha}} \\
& =\sum_{n \geq 0} x^{n} \sum_{\alpha \vdash n} e^{y\left(\sum_{\beta \vdash r} \prod_{i=1}^{r}\left(\begin{array}{c}
a_{i} \\
b_{i}
\end{array}\right)\right)} \frac{1}{z_{\alpha}} \quad(\text { by } 6.12) \\
& =\sum_{n \geq 0} x^{n} \sum_{a_{1}+2 a_{2}+\cdots+n a_{n}=n} e^{y\left(\sum_{\beta \vdash r} \prod_{i=1}^{r}\left(\begin{array}{c}
a_{i} \\
b_{i}
\end{array}\right)\right)} \frac{1}{1^{a_{1}} a_{1} ! 2^{a_{2}} a_{2} ! \cdots n^{a_{n}} a_{n} !} \\
& =\left.\sum_{n \geq 0} x^{n}\left(\sum_{a_{1} \geq 0} \sum_{a_{2} \geq 0} \cdots \sum_{a_{n} \geq 0} \frac{1}{a_{1} !}\left(\frac{x}{1}\right)^{a_{1}} \frac{1}{a_{2} !}\left(\frac{x^{2}}{2}\right)^{a_{2}} \cdots \frac{1}{a_{n} !}\left(\frac{x^{n}}{n}\right)^{a_{n}} e^{y\left(\sum_{\beta \vdash r} \prod_{i=1}^{r}\left(\begin{array}{c}
a_{i} \\
b_{i}
\end{array}\right)\right)}\right)\right|_{x^{n}} \\
& =\left.\sum_{n \geq 0} x^{n}\left(\frac{e^{-\frac{x}{1}-\frac{x^{2}}{2}-\cdots-\frac{x^{r}}{r}}}{1-x} \sum_{a_{1} \geq 0} \cdots \sum_{a_{n} \geq 0} \frac{1}{a_{1} !}\left(\frac{x}{1}\right)^{a_{1}} \cdots \frac{1}{a_{n} !}\left(\frac{x^{n}}{n}\right)^{a_{n}} e^{y\left(\sum_{\beta \vdash r} \prod_{i=1}^{r}\left(\begin{array}{c}
a_{i} \\
b_{i}
\end{array}\right)\right)}\right)\right|_{x^{n}} \\
& =\frac{e^{-\frac{x}{1}-\frac{x^{2}}{2}-\cdots-\frac{x^{r}}{r}}}{1-x} \sum_{a_{1} \geq 0} \cdots \sum_{a_{r} \geq 0} \frac{1}{a_{1} !}\left(\frac{x}{1}\right)^{a_{1}} \cdots \frac{1}{a_{n} !}\left(\frac{x^{n}}{n}\right)^{a_{n}} e^{y\left(\sum_{\beta \vdash r} \prod_{i=1}^{r}\left(\begin{array}{c}
a_{i} \\
b_{i}
\end{array}\right)\right) .}
\end{aligned}
$$

This proves equation 6.22 and completes our argument.

The special cases $r=2,3,4$ of 6.22 reduce to

$$
\begin{aligned}
& (1-x) F_{2}(x, y)=e^{-x} e^{\frac{x^{2}}{2}\left(e^{y}-1\right)} \sum_{a_{1} \geq 0} \frac{x^{a_{1}}}{a_{1} !} e^{y\left(\begin{array}{c}
a_{1} \\
2
\end{array}\right)} \\
& (1-x) F_{3}(x, y)=e^{-x} e^{\frac{x^{3}}{3}\left(e^{y}-1\right)} \sum_{n \geq 0} \frac{x^{n}}{n !} e^{y\left(\begin{array}{c}
n \\
3
\end{array}\right)+\frac{x^{2}}{2}\left(e^{y n}-1\right)} \\
& (1-x) F_{4}(x, y)=\frac{e^{\frac{x^{4}}{4}\left(e^{y}-1\right)}}{e^{x+x^{2} / 2}} \sum_{a_{1} \geq 0} \sum_{a_{2} \geq 0} \frac{\left(\frac{x}{1}\right)^{a_{1}} !}{a_{1} !} \frac{\left(\frac{x^{2}}{2}\right)^{a_{2}}}{a_{2} !} e^{y\left(\begin{array}{c}
a_{1} \\
4
\end{array}\right)+y\left(\begin{array}{c}
a_{1} \\
2
\end{array}\right) a_{2}+y\left(\begin{array}{c}
a_{2} \\
2
\end{array}\right)} e^{\frac{x^{3}}{3}\left(e^{y a_{1}}-1\right)}
\end{aligned}
$$

From equation 6.19 it follows that the stable values of the Kronecker coefficient $\left\langle\left(h_{r} h_{n-r}\right)^{* k}, h_{n}\right\rangle$ counts coverings of the sets $\Omega_{n}$ with $k$ sets of size $r$ so that every $x \in \Omega_{n}$ belongs to at least one set. Thus the generating function in equation 6.22 may 
be viewed as a solution of an enumeration problem first formulated by Comtet in [Co]. It is interesting to note that for $r=1,\left\langle\left(h_{1} h_{n-1}\right)^{* k}, h_{n}\right\rangle$ reduces to the Bell number $B_{k}$. Thus using character polynomials we derive that

$$
B_{k}=\sum_{\alpha=1^{a_{1} \ldots k^{a} k \vdash k}} \frac{a_{1}^{k}}{z_{\alpha}}
$$

A formula that does not seem to exist in the literature. In the Labelle case $r=2$, the stable value of $\left\langle\left(h_{2} h_{n-2}\right)^{* k}, h_{n}\right\rangle$ counts of the number $\omega_{k}$ of multigraphs with $k$ edges and no loop, where every vertex belongs to at least one edge. Thus using character polynomials we derive that

$$
\omega_{k}=\sum_{\alpha=1^{a_{1}} \ldots(2 k)^{a_{2 k} \vdash 2 k}} \frac{\left(a_{2}+\left(\begin{array}{c}
a_{1} \\
2
\end{array}\right)\right)^{k}}{z_{\alpha}}
$$

The cases $r \geq 3$ does not seem to have been treated in the literature. For example when $r=3$, the sets $\Omega_{m}$ are covered with $k$ triangles so that every $x \in \Omega_{m}$ belongs to at least one triangle, etc. Here is a table of some stable values of $\left\langle\left(h_{r} h_{n-r}\right)^{* k}, h_{n}\right\rangle$ :

\begin{tabular}{|l|l|l|l|l|l|l|l|l|l|}
\hline$k$ & 1 & 2 & 3 & 4 & 5 & 6 & 7 & 8 & 9 \\
\hline$r=1$ & 1 & 2 & 5 & 15 & 52 & 203 & 877 & 4140 & 21147 \\
$r=2$ & 1 & 3 & 16 & 139 & 1750 & 29388 & 624889 & 16255738 & 504717929 \\
$r=3$ & 1 & 4 & 39 & 862 & 35775 & 2406208 & 238773109 & 32867762616 & 6009498859909 \\
\hline
\end{tabular}

Table 6.1 Multiplicity of $h_{n}$ in the $k^{\text {th }}$ Kronecker power of $h_{r} h_{n-r}$ for $r=1,2,3$

As our final applications, we give the expressions of some special character polynomials and their combinatorial interpretations.

Proposition 6.1 For positive integers $k$ and partitions $\mu \vdash k$, let $f_{\mu}=\chi_{1^{k}}^{\mu}$ be the number of standard tableaux of shape $\mu$. Then the polynomials $g_{k}(x)=\sum_{\mu \vdash k} f_{\mu} q_{\mu}(x)$ depend only on the variable $x_{1}$ and are given by the identity

$$
\sum_{\mu \vdash k} f_{\mu} q_{\mu}(x)=\sum_{r=0}^{k}(-1)^{k-r}\left(\begin{array}{l}
k \\
r
\end{array}\right)\left(x_{1}\right)_{r}
$$

and they satisfy the following recurrence of Laguerre polynomials:

$$
g_{k}\left(x_{1}\right)=g_{k}\left(x_{1}-1\right)+k g_{k-1}\left(x_{1}-1\right)
$$

THE ELECTRONiC Journal of COMBinatorics 16(2) (2009), \#R19 
Proof. We use the fact that the $k$ th power $e_{1}^{k}$ of the elementary symmetric polynomial $e_{1}$ can written as $e_{1}^{k}(x)=\sum_{\mu \vdash k} f_{\mu} s_{\mu}(x)$. Using (2.12) we have

$$
q_{e_{1}^{k}}(x)=\sum_{\mu \vdash k} f_{\mu} q_{\mu}(x)
$$

On the other hand from equation I.5 we have $q_{e_{1}^{k}}(x)=\downarrow\left(x_{1}-1\right)^{k}$. This then gives $\sum_{\mu \vdash k} f_{\mu} q_{\mu}(x)=\sum_{r=0}^{k}(-1)^{k-r}\left(\begin{array}{l}k \\ r\end{array}\right)\left(x_{1}\right)_{r}$ which is identity 6.23 . The fact that the right hand side of identity 6.23 satisfies the recurrence 6.24 can be verified directly.

An immediate consequence of identity 6.25 is that when $\alpha=1^{n}$, then $\chi_{1^{n}}^{(n-k, \mu)}=$ $f_{(n-k, \mu)}$ is the number of standard tableaux of shape $(n-k, \mu)$ and:

$$
q_{e_{1}^{k}}(\alpha)=q_{e_{1}^{k}}(n)=\sum_{\mu \vdash k} f_{\mu} f_{(n-|\mu|, \mu)} .
$$

The right hand side of equation 6.26 is counting pairs $(P, Q)$ of standard tableaux of respective weights $k$ and $n$. If we add a row of length $n-k$ to the left tableau $P$ and fill it with the numbers $1,2, \ldots, n-k$, then we obtain a pair of standard tableaux of the same weigth $n$ that corresponds to a permutation via the Robinson-Schensted correspondance and the following interpretation is derived:

Corollary 6.1 For every positive integer $k$, the character polynomial $q_{e_{1}^{k}}(n)$ counts the number of pairs of standard tableaux $(P, Q)$ of same shape where the first row of the tableau $P$ is filled with the numbers $1,2, \ldots, n-k$.

Corollary 6.2 For every positive integers $k, n$ with $n \geq 2 k$ the polynomial $f_{k}(n)=\sum_{r=0}^{k}\left(\begin{array}{l}k \\ r\end{array}\right)(-1)^{r} n(n-1) \cdots(n-k+r+1)$ counts the number of permutations $\sigma \in S_{n}$ with longest increasing subsequence $\sigma_{1}<\sigma_{2}<\cdots<\sigma_{n-k}=n$.

Proof. Exchange the roles of $P$ and $Q$ in the previous corollary and use the reverse Robinson-Schensted correspondance.

For example the permutation $\sigma=12543$ has three increasing subsequences of length 3 and is an admissible permutation when $k=2, n=5$ and $f_{2}(5)=11$. Interestingly, we found no elementary combinatorial proof of this simple identity. The following character polynomials are easily derived from standard symmetric functions identities. 
Proposition 6.2 For all integers $k \geq 0$ we have
a) $\sum_{i=0}^{k-1}(-1)^{i} q_{\left(k-i, 1^{i}\right)}(x)=k x_{k}-1$
b) $q_{\left(1^{k}\right)}(x)=\sum_{\alpha \vdash k}\left(\begin{array}{l}k \\ r\end{array}\right)\left(\begin{array}{c}x_{1}-1 \\ a_{1}\end{array}\right)(-1)^{k-\ell(\alpha)} \prod_{i=2}^{k}\left(\begin{array}{c}x_{i} \\ a_{i}\end{array}\right)$
c) $q_{\left(1^{k}\right)}(x)+q_{\left(1^{k-1}\right)}(x)=\sum_{\alpha \vdash k}(-1)^{\operatorname{sgn}(\alpha)} \prod_{i=1}^{k}\left(\begin{array}{c}x_{i} \\ a_{i}\end{array}\right)$
d) $q\left(h_{r} h_{n-r}\right)=\sum_{i=0}^{r} q\left(s_{(i)}\right)=\sum_{\alpha \vdash r} \prod_{i=1}^{r}\left(\begin{array}{c}x_{i} \\ a_{i}\end{array}\right)$

\section{References.}

[CT] C. Carre, J.Y. Thibon, Plethysm and Vertex operators, Adv. Appl. Math. vol 13 no 4, 73, 390-403, 1992.

[Co] L. Comtet, Analyse combinatoire, Presses universitaires de France, Paris, 1970.

[GC] A. Goupil, C. Chauve, Combinatorial decomposition of Kronecker powers of irreducible representations of $S_{n}$, Séminaire Lotharingien de Combinatoire, 54, 2006, article B54J.

[Ke] A. Kerber, Applied Finite Group Actions, Second Edition. Springer Verlag, 1999.

[La] G. Labelle, Counting enriched multigraphs according to the number of their edges (or arcs), Discrete Mathematics, vol 217, no 1, pp 237-248, 2000.

[Li] D.E. Littlewood, The Kronecker product of symmetric group representations, J. London Math. Soc. 31, 1956, 89-93.

[Ma] I.G. Macdonald, Symmetric Functions and Hall Polynomials, Oxford U. press, second edition, 1995.

[Mu] F.D. Murnaghan, The Analysis of the Kronecker product of Irreducible Representations of the Symmetric Group, American Journal of Mathematics, vol. 60 no 3, pp 761-784, 1938.

[Ra] A. Ram, A Frobenius formula for the characters of the Hecke algebras, Inventiones Mathematica, vol 106, no 1, pp 461-488, 1991.

[Sp] W. Specht, Die Charactere der symmetrischen Gruppe, Math. Zeitschr. 73, $312-329,1960$.

[Va] E. Vallejo, Stability of Kronecker products of irreducible characters of the symmetric group, Electron. J. Combin. 6, Research Paper 39, 7 pp.,1999.

[WW] E. T. Whittaker, G. N. Watson, A Course of Modern Analysis, Cambridge U. Press, 4th edition, 1927. 